\title{
An interior point method for isogeometric contact
}

\author{
İ. Temizer ${ }^{\mathrm{a}, *}$, M.M. Abdalla ${ }^{\mathrm{b}}, \mathrm{Z}$. Gürdal ${ }^{\mathrm{c}}$ \\ a Department of Mechanical Engineering, Bilkent University, 06800 Ankara, Turkey \\ ${ }^{\mathrm{b}}$ Faculty of Aerospace Engineering, Delft University of Technology, Kluyverweg 1, 2629 HS Delft, The Netherlands \\ ${ }^{\mathrm{c}}$ Department of Mechanical Engineering, University of South Carolina, Columbia, SC 29208, United States
}

Received 20 November 2013; received in revised form 10 March 2014; accepted 27 March 2014

Available online 30 April 2014

\begin{abstract}
The interior point method is applied to frictionless contact mechanics problems and is shown to be a viable alternative to the augmented Lagrangian approach. The method is derived from a mixed formulation which induces a contact discretization scheme in the spirit of the mortar method and naturally delivers slack variables that help constrain the solution to the feasible region. The derivation of the algorithm as well as its robustness benefits from the contact interface description that is induced by NURBS-based isogeometric volume discretizations. Various interior point algorithms are discussed, including a primal-dual approach that satisfies the unilateral contact constraints exactly, in addition to two primal approaches that retain an arbitrary barrier parameter. The developed algorithms can easily be pursued starting from an augmented Lagrangian implementation. Numerical investigations on benchmark problems demonstrate the efficiency and the robustness of the framework, but also highlight current limitations that suggest paths for future research. Overall, the results indicate that the interior point method can challenge the augmented Lagrangian method in contact mechanics, even displaying potential for higher efficiency and robustness.
\end{abstract}

(C) 2014 Elsevier B.V. All rights reserved.

Keywords: Contact; Interior point method; Mortar discretization; Isogeometric analysis

\section{Introduction}

Mathematically, contact poses an optimization problem with inequality constraints. It is therefore no surprise that the augmented Lagrangian (AL) method [1] has gradually established itself as the most favorable method for solving contact mechanics problems with simultaneous efficiency and robustness, since it is one of the most favored methods for nonlinear optimization problems as well. However, recent advances suggest the interior point (IP) method to be a viable alternative — see [2] for an extensive review. In its simplest form, the IP method is the classical barrier method $[3,4]$. The form of the barrier function can be physically

\footnotetext{
* Corresponding author. Tel.: +90 (312) 290 3064; fax: +90 (312) 2664126.

E-mail address: temizer@bilkent.edu.tr (I. Temizer).
} 
motivated by microscopic contact mechanisms [5,6]. From an optimization point of view, on the other hand, the barrier is classically logarithmic. Independent of the particular choice, a straightforward application of any barrier function faces challenges, for instance due to the need to prevent the penetration of the surfaces in order to avoid an unphysical interface pressure. The choices made in addressing these challenges directly impact the efficiency and the robustness of the IP method. Indeed, early comparison studies indicated that the AL approach is more robust [7]. In this work, it will be demonstrated that the IP method can challenge the AL approach, even displaying potential for higher efficiency and robustness.

In comparison to penalty, Lagrange multiplier and AL methods, there is only a limited number of studies concentrating on the application of the IP method in its modern form to contact mechanics. The work [7] already considered Coulomb friction and additionally introduced slack variables that are crucial to effectively constraining the solution to the feasible region — see also [8] for similar studies without friction. A solution scheme that is reminiscent of Uzawa staggering for the multiplier updates in the AL approach has been considered in [9] for frictionless contact, also using slack variables. Both of the works are in a two-dimensional setting, the former limited to linear elasticity and a single deformable body while the latter considers hyperelasticity with two deformable bodies and non-matching meshes based on a node-to-segment strategy for formulating contact. A hybrid method based on a combination of the AL and the IP methods was proposed in [10] for the linearly elastic frictionless contact of two bodies with matching meshes in a node-to-node setting. Kučera et al. [11] considers a linearly elastic three-dimensional body in contact with a rigid obstacle, with Coulomb as well as Tresca friction. One can also identify applications in the optimization literature, for instance $[12,13]$. While all of these works are based on the finite element method, the boundary element method can also be an efficient choice for large scale linear elasticity problems with contact [14].

There are two aspects to investigating an IP method for contact problems. The optimization aspect is still an active research area. The algorithm employed in this work is an established framework yet leaves room for improvement. For all aspects of the IP method from an optimization perspective, from the terminology to the methods chosen, [15] will be made use of extensively and without further reference unless needed for clarity. See also [16] for an overview. The second aspect concerns the discretization of the problem, both that of the bodies as well as the contact variables. To some extent, the optimization algorithm can be varied for a given discretization method, although the particular method will strongly affect the overall robustness of the framework. Presently, the aim is to develop and test a strategy that is suitable for three-dimensional, frictionless finite deformation contact problems with non-matching meshes. The mortar method has established itself as the most robust contact discretization approach in this context and will be made use of in this work detailed references will be provided in upcoming sections. The discretization of the volume interacts naturally with the contact discretization. Here, NURBS-based isogeometric analysis will be employed, initiated in [17] and reviewed in [18]. Recent applications include porous media modeling [19], boundary element analysis [20], fluid mechanics [21] and shells [22] with optimization [23]. Isogeometric contact approaches, where a smooth surface finite element discretization is naturally induced by the volume discretization, were initiated in [24,25]. Various advantages reported in recent works will be highlighted. Nevertheless, to the best knowledge of the authors', the present work also constitutes a first attempt towards combining IP methods with the mortar idea. Therefore, although not pursued in this work, the use of mortar formulations developed on the basis of Lagrange discretizations would be equally interesting.

The work is organized as follows. In Section 2, the basic barrier regularization idea behind the IP method is reviewed. The mixed formulation basis of the regularized formulation is presented, where slack variables naturally appear and provide a means for avoiding an unphysical interface pressure. The mixed formulation additionally induces a contact discretization scheme in the spirit of the mortar method, as outlined in Section 3. In particular, a lumped formulation that is convenient for numerical implementation will be derived through a series of simplifying assumptions. A primal-dual strategy is then discussed in Section 4 where the aim is to reduce the barrier parameter towards zero. This is realized within a two-stage predictor-corrector scheme embedded in an iteration of the Newton-Raphson method for solving the nonlinear problem. Alternative primal formulations will additionally be introduced as simplifications of the primal-dual strategy. Numerical investigations will be provided in Section 5 where two-body frictionless elastic contact problems will be discussed and comparisons with the AL method will be carried out. 


\section{Interior point method}

\subsection{Regularization}

The contribution to the weak form of the linear momentum balance due to frictionless contact is

$$
\delta \mathcal{G}^{c}=-\langle\delta g p\rangle .
$$

Here, $p \geqslant 0$ is the referential contact pressure and, within a classical slave/master setting,

$$
g=-(\boldsymbol{x}-\boldsymbol{y}) \cdot \boldsymbol{v}
$$

is the normal gap with $\boldsymbol{x}$ as the current position of a point on the slave surface, $\boldsymbol{y}$ refers to its closest-point projection on the master and $v$ is the outward unit normal to the current configuration of the master surface at point $\boldsymbol{y}$. For compactness, the notation

$$
\langle\bullet\rangle=\int_{\Gamma_{o}^{c}} \bullet \mathrm{d} A
$$

has been adopted. The reference configurations of all slave surfaces that could potentially come into contact with the master constitute the domain of integration $\Gamma_{o}^{c}$. When there is no contact on a portion of the slave surface, $p$ is zero so that the contribution to the integral vanishes. For a detailed overview of contact kinematics, the reader is referred to $[26,27]$.

Physically, the contact contribution is governed by the unilateral contact constraints

$$
g \leqslant 0, \quad p \geqslant 0, \quad g p=0 .
$$

In a typical exterior point (EP) method [5], these constraints are relaxed by allowing the solution to leave the feasible region $g \leqslant 0$. The violation $g>0$ is then penalized by a penalty parameter $\epsilon>0$, leading to the regularized model

$$
p=\epsilon g \text {. }
$$

In an algorithmic setting, this exterior penalty approach intrinsically involves an active set — only points for which $g>0$ are considered. The penalty parameter is increased to drive the active points towards the boundary of the feasible region $(g=0)$.

In an interior point method, on the other hand, one attempts to keep the solution within the feasible region. A suitable regularization for this purpose is a barrier formulation [26]

$$
p=-\mu / g \text {. }
$$

There is no active set in this IP method - all points are active at all times. By decreasing the barrier parameter $\mu>0$, the solution is driven to the boundary of the feasible region. The algorithmic challenge in the application of this approach lies in ensuring that points remain within the feasible region $g<0$ to avoid undefined or negative pressures. Within a typical Newton-Raphson type iterative solution of a contact problem based on this method, the incremental update may move the solution outside the feasible region and there is no straightforward procedure for modifying the displacement update to prevent this violation. This is a central challenge.

\subsection{Mixed formulation}

In order to meet the algorithmic challenge associated with the use of $g$ in the IP method, a variational formulation can be introduced. For this purpose, it is useful to recall the classical three-field mixed variational formulation of penalty-regularized normal contact, introduced in [28]. The starting point of this mixed formulation is to define a contact functional $\mathcal{C}$ by adding a mixed kinematic variable $\gamma$ into the formulation. The classical penalty contact potential which delivers the regularized form of (2.1) upon variation reads

$$
\mathcal{E}[g]=-\frac{\epsilon}{2}\left\langle g^{2}\right\rangle
$$


The mixed formulation defines

$$
\mathcal{C}[g, \gamma, p]=\mathcal{E}[\gamma]-\langle p(g-\gamma)\rangle .
$$

When added to the functional describing the bulk response, the variation of $\mathcal{C}$ delivers the classical contribution (2.1) to the weak form as well as the two weak relations

$$
\langle\delta p(g-\gamma)\rangle=0 \quad \text { and } \quad\langle\delta \gamma(p-\epsilon \gamma)\rangle=0 .
$$

When all variables have infinite degrees of freedom, i.e. in the continuum setting, these relations enforce $\gamma=g$ and hence $p=\epsilon g$, thereby recovering the classical EP method. Consequently, in a continuum setting there is no apparent advantage of reverting to a mixed formulation for the EP method. The advantage appears when the problem is discretized - see also Section 3. The situation is slightly different for the IP method. The classical barrier contact potential reads

$$
\mathcal{I}[g]=\mu\langle\ln |g|\rangle
$$

and results in the algorithmic challenge of ensuring $g<0$. Instead, following the idea in [28], a variational formulation can be pursued by introducing an additional mixed kinematic variable $s$ based on the functional

$$
\mathcal{C}[g, s, p]=\mathcal{I}[s]-\langle p(g+s)\rangle .
$$

As for the EP method, the variation of this functional delivers the contribution (2.1) as well as the two weak relations

$$
\langle\delta p(g+s)\rangle=0 \quad \text { and } \quad\langle\delta s(p-\mu / s)\rangle=0,
$$

such that in the continuum setting one assigns $s=-g$ and therefore $p=-\mu / g$. Alternatively, however, one may refrain from this assignment and retain $s$ as an independent variable. In this setting, the pressure is evaluated via the original implication of the weak relations:

$$
p=\mu / s \text {. }
$$

Unlike $g, s$ is not directly linked to the kinematics of the contacting bodies and hence it is possible to guarantee $s>0$. Therefore, the mixed formulation provides a means for circumventing the difficulty associated with the use of $g$, hence already displaying an advantage without discretization. $s$ is referred to as a slack variable in the optimization literature. The algorithm for updating the value of $s$ will be introduced in 4.3. In what follows, $s>0$ is assumed.

\section{Mortar-based contact discretization}

\subsection{Integration point and node-to-surface methods}

In this section, a novel finite element discretization of the IP method will be introduced. Clearly, the simplest approach to the implementation of the IP method follows its EP counterpart in a continuum setting, starting from the discretization of the slave and master surfaces

$$
\boldsymbol{x}=\sum_{I} N^{I} \boldsymbol{x}^{I}, \quad \boldsymbol{y}=\sum_{J} M^{J} \boldsymbol{y}^{J}
$$

Here, $N^{I}$ are the shape functions of the slave and $M^{J}$ are those of the master. At any stage of the solution procedure, the surface discretizations deliver the value of $g$, and indirectly $s$, at each integration point of the slave elements throughout the potential contact interface via (2.2), thereby enabling the integration of the contact contribution to the weak form either via the EP or the IP method. In the context of the EP method, it is well known that this approach leads to surface locking [29,30], leading to unphysical solutions as $\epsilon$ is increased. Not surprisingly, the IP algorithm displays the same problem, leading to unphysical solutions as $\mu$ is decreased.

The alternative to the integration point approach outlined above is the classical node-to-surface algorithm where $g$ is calculated only at the nodes of the slave surface through their projections onto the master [26,27]. Therefore, it is possible to update $s$ at the nodes $[7,9,8]$. However, several problems have been identified with 
this approach in the context of the EP method. First, while the integration point approach passes the contact patch test, the node-to-surface algorithm in its simplest form fails it $[28,31,32]$. Second, since contact interactions are associated with nodal locations only, sharp changes in the contact forces are often observed as nodes change their contact status which may lead to numerical convergence problems $[33,34]$. Third, control points play the role of nodes in isogeometric analysis but since isogeometric discretizations are not interpolatory the gap associated with the location of a control point is not physical, leading to difficulties in the construction of a node-to-surface type algorithm $[24,25]$ — see [35] for a recent collocation approach in this context.

\subsection{Mortar method}

The mortar method circumvents all of the difficulties mentioned above. In particular, it passes the contact patch test and does not display surface locking. Developed originally for domain decomposition with nonmatching interface meshes [36-38], the mortar method has extensively been applied to contact problems without $[39,33,40-44]$ and with friction $[45,34,46-51]$. However, all of these applications have exclusively been in the context of the EP penalty (primal) $[45,46,40,47]$ and Lagrange multiplier (dual, with a slight abuse of terminology since a dual problem is not constructed) $[39,40,52,51,44]$ methods or combinations thereof, such as the AL (primal-dual) method [41,42,48,49,43] (possibly in a staggered form with Uzawa iterations $[33,34,50,53])$. In this section, the IP method will be discretized in the spirit of the mortar method.

Within the numerical investigations, comparisons of the primal and primal-dual formulations of the IP method with both the AL and the penalty methods will eventually be carried out in a mortar setting. Roughly speaking, the iterative convergence characteristics for the primal-dual IP and EP formulations will be observed to be similar, as is the case for the primal IP and EP formulations. Primal-dual formulations satisfy the discrete contact constraints exactly whereas the regularization parameters in the primal formulations must be chosen judiciously for a satisfactory solution. Although the former are computationally slightly more expensive due to the additional presence of multipliers, they typically display a significantly more robust behavior. It is remarked that the form of the AL method to be employed in this work follows [1] where the multiplier and augmentation contributions to the contact pressure are linearized within a monolithic scheme. The alternative Uzawa-staggered form is less efficient and its convergence is strongly affected by the choice of the penalty parameter. Even when strategies are adopted to update the penalty parameter through Uzawa iterations [26], its performance remains inferior to the monolithic form.

It is also remarked that mortar methods are typically accompanied by accurate integration algorithms for the evaluation of the contact integrals. This is accomplished through the use of an intermediate integration surface, suggested as early as [54] but the construction of which was introduced in [37] for three-dimensional finite deformation problems. A strategy for introducing an intermediate surface for mortar-based isogeometric domain decomposition techniques was recently developed in [55]. Presently, following [40] and consistent with the notation employed, the integration surface is retained as the slave surface with the implication that (i) the contact patch test will not be passed to machine precision even for flat interfaces and (ii) some degree of robustness loss may be observed in the numerical algorithm. Nevertheless, this approach leads to considerable ease in implementation and it has been shown to deliver satisfactory robustness and accuracy in a broad range of contact problems in the context of isogeometric analysis.

The derivation of a suitable mortar approach can be conveniently based on the mixed formulation (2.11). Such a construction, implicit in the construction of all mortar methods (see [39] for an early example), was demonstrated in the context of the EP method without [56] and with friction [53]. Applying the core idea of the mortar method in the form adopted in [33], it is proposed that not only the pressure but also the slack variables are discretized using the slave discretization:

$$
p=\sum_{I} N^{I} p^{I}, \quad s=\sum_{I} N^{I} s^{I} .
$$

Although the same order of discretization is employed for the displacement and contact degrees of freedom, earlier works on isogeometric contact have not detected any stability issues with this choice. Specifically, the local pressure distribution and the global loads converge with mesh refinement [57,58]. Additionally, the patch test is satisfied without displaying surface locking [53]. 
The first of the weak relations in (2.12) now implies

$$
\sum_{I} \delta p^{I}\left\langle N^{I}(g+s)\right\rangle=0
$$

or

$$
\left\langle N^{I} s\right\rangle=-\left\langle N^{I} g\right\rangle \text {. }
$$

The physical gap $g$ does not admit a discretization but $s$ does, leading to a linear system of equations that deliver the discrete values of $s$ :

$$
\sum_{J}\left\langle N^{I} N^{J}\right\rangle s^{J}=-\left\langle N^{I} g\right\rangle
$$

Consequently, $s$ is not necessarily equal to $g$ pointwise but only in the weighted sense in (3.4). It is recalled that $s^{I}$ must additionally be subjected to a positivity requirement to avoid undefined or unphysical pressures - see Section 4.3 .

The second of the weak relations in (2.12) implies

$$
\sum_{I} \delta s^{I}\left\langle N^{I}(p-\mu / s)\right\rangle=0
$$

or

$$
\left\langle N^{I} p\right\rangle=\mu\left\langle N^{I} / s\right\rangle,
$$

thereby leading to a linear system of equations for the discrete values of $p$ provided $s$ has already been determined:

$$
\sum_{J}\left\langle N^{I} N^{J}\right\rangle p^{J}=\mu\left\langle N^{I} / s\right\rangle
$$

\subsection{Lumped formulation}

The results (3.5) and (3.8) are inconvenient for two reasons. First, they require the solution of linear systems of equations. Second, the discrete values of $s$ cannot be moved out of the integral expression on the right-hand side of (3.8), which is inconvenient for the numerical implementation. The first of these can be resolved through a simple lumping approach — see [56] for alternative approaches in the context of the present derivation. For (3.5) this results in

$$
s^{I}=-\frac{\left\langle N^{I} g\right\rangle}{\left\langle N^{I}\right\rangle} .
$$

Here, an important feature of isogeometric discretizations comes into play, namely the non-negativity of the basis functions [18]. This feature guarantees that $s^{I}$ is positive whenever the physical gap $g$ is negative throughout the interface. This is not true for discretizations based on the classical Lagrange basis functions, leading to complications for higher-order mortar formulations $[42,47,50]$. Presently, the same mortar algorithm will be uniformly applicable to all orders of isogeometric (NURBS) discretizations without any special modification [58-60], thereby simplifying the treatment of geometries with varying orders of discretizations.

For (3.8), on the other hand, it is desirable to apply lumping to the left-hand side and also simplify the right-hand side. In relation to the continuum expression (2.13), obtaining the result

$$
p^{I}=\mu / s^{I}
$$

would prove convenient whereas lumping alone delivers

$$
p^{I}=\mu \frac{\left\langle N^{I} / s\right\rangle}{\left\langle N^{I}\right\rangle} \text {. }
$$


Hence, the simplification of the right-hand side must be justified. This is possible through energy arguments. The contribution to the weak form is, upon repeatedly using (3.2), (3.4) and (3.7),

$$
\begin{aligned}
\delta \mathcal{G}^{c} & =-\langle\delta g p\rangle=-\sum_{J}\left\langle\delta g N^{J}\right\rangle p^{J}=\sum_{J}\left\langle\delta s N^{J}\right\rangle p^{J} \\
& =\langle\delta s p\rangle=\sum_{I}\left\langle N^{I} \delta s^{I} p\right\rangle=\sum_{I} \delta s^{I}\left\langle N^{I} p\right\rangle=\sum_{I} \mu \delta s^{I}\left\langle N^{I} / s\right\rangle \\
& =\mu\langle\delta s / s\rangle=\mu \delta\langle\ln |s|\rangle=\delta \mathcal{I}[s] .
\end{aligned}
$$

For insufficiently small values of $\mu,-\mathcal{G}^{c}=-\mathcal{I}[s]>0$ represents a convex interface energy. Since $s^{I}$ will be retained in the feasible (positive) region and the isogeometric basis functions satisfy the partition of unity, Jensen's inequality guarantees

$$
-\mathcal{I}[s]=-\mu\langle\ln |s|\rangle \leqslant-\mu \sum_{I}\left\langle N^{I} \ln \left|s^{I}\right|\right\rangle=-\widetilde{\mathcal{I}}[s] .
$$

Consequently, the minimization of $-\widetilde{\mathcal{I}}$ will ensure the minimization of $-\mathcal{I}$. Specifically, if $-\widetilde{\mathcal{I}}$ is driven to zero by decreasing $\mu$ towards zero then $-\mathcal{I}$ is guaranteed to be driven to zero. In this sense, $\widetilde{\mathcal{I}}$ is a suitable approximation of $\mathcal{I}$. When $\widetilde{\mathcal{I}}$ is used in the mixed formulation (2.11) instead of $\mathcal{I}$, followed by lumping, one ends up with (3.10) as desired instead of (3.11).

Above, it was assumed that the same $s^{I}$ values are used to evaluate both $\mathcal{I}$ and $\widetilde{\mathcal{I}}$. Clearly, the original and approximate formulations will deliver different $s^{I}$ values such that the inequality in (3.13) may not hold. Nevertheless, the numerical investigations in Section 5.2 will demonstrate that the difference between the two potentials remains very small which suggests that the solutions obtained from both formulations would probably also be very close. In view of this observation, the algorithmic convenience of the approximation is very appealing.

To summarize, the final discrete form of the functional (2.11) from which the mortar-based IP method emanates is

$$
\mathcal{C}=\sum_{I}\left\{\mu w^{I} \ln s^{I}-p^{I}\left(\bar{g}^{I}+w^{I} s^{I}\right)\right\}
$$

where the notation

$$
w^{I}=\left\langle N^{I}\right\rangle, \quad \bar{g}^{I}=\left\langle N^{I} g\right\rangle
$$

has been introduced for further notational compactness in upcoming algorithmic discussions.

\section{Primal-dual strategy}

\subsection{Gap and complementarity conditions}

The IP method introduced so far is a primal strategy where the contribution to the weak form of the linear momentum balance is determined by a mortar-based discretization

$$
\delta \mathcal{G}^{c}=-\sum_{I} \delta \bar{g}^{I} p^{I}
$$

with the lumped formulation $p^{I}=\mu / s^{I}$ where $s^{I}$, ideally equal to $-\bar{g}^{I} / w^{I}$, will be algorithmically ensured to be positive. It is remarked that, since lumping is not applied to the contact contribution, the analysis presented in $[33,44]$ regarding momentum conservation is applicable to the present formulation as well. Specifically, when the problem demands it, the linear momentum will be conserved provided the mortar integrals are evaluated exactly.

Provided $\mu$ is chosen sufficiently small, a satisfactory solution is expected. However, an appropriate choice of $\mu$ may not always be straightforward. A primal-dual strategy that is also based on the functional (3.14) may be constructed to circumvent this problem wherein $s^{I}$ will be driven to zero at the contact interface and $p^{I}$ will be regarded as independent Lagrange multipliers. Note that this needs to be realized by driving $\mu$ to zero since 
the barrier term in (3.14) would otherwise be undefined. The method of determining how $\mu$ is decreased dictates the solution scheme. Here, one common scheme is adopted from the optimization literature. Extensive discussions on the apparent ill-conditioning that arises as $\mu \rightarrow 0$ as well as methods of circumventing it may be found in $[15,16]$.

The starting point for a primal-dual approach is to restate the lumped formulations as

$$
w^{I} s^{I}+\bar{g}^{I}=0, \quad s^{I} p^{I}=\mu,
$$

the former to be referred to as the gap and the latter as the complementarity condition. Within the primal setting, the former condition is satisfied only at convergence, due to the necessity of ensuring $s^{I}>0$. The latter, however, is enforced directly. In the primal-dual setting, both of these conditions are satisfied only at convergence. Their linearizations therefore deliver

$$
w^{I} s^{I}+\bar{g}^{I}+w^{I} \Delta s^{I}+\Delta \bar{g}^{I}=0, \quad s^{I} p^{I}+\Delta s^{I} p^{I}+s^{I} \Delta p^{I}+\Delta s^{I} \Delta p^{I}=\mu
$$

with the non-incremental terms not necessarily adding to zero. Note the presence of the seemingly unfitting quadratic term $\Delta s^{I} \Delta p^{I}$. This term will be initialized to zero and then recomputed in a predictor-corrector algorithm in the primal-dual strategy. If the quadratic term is omitted then one essentially obtains a scheme that is equivalent to the primal one - see Section 4.5. Its presence serves two purposes: (i) it helps to quicker satisfy the complementarity with the updated solution, i.e. $\left(s^{I}+\Delta s^{I}\right)\left(p^{I}+\Delta p^{I}\right)=\mu$, and (ii) it delivers an estimate of $\mu$ for the next iteration.

$\Delta s^{I}$ may be isolated from the latter result in (4.3) as

$$
\Delta s^{I}=\left(\mu-s^{I} p^{I}-s^{I} \Delta p^{I}-\Delta s^{I} \Delta p^{I}\right) / p^{I}
$$

and then substituted into former to yield, after rearrangement,

$$
\Delta \bar{g}^{I}-w^{I} s^{I} \Delta p^{I} / p^{I}=-w^{I}\left(\mu-\Delta s^{I} \Delta p^{I}\right) / p^{I}-\bar{g}^{I} .
$$

Recalling that the gap condition emanates from the (lumped) weak statement (3.3), this result may be restated in weak form as

$$
\sum_{I} \delta p^{I}\left(\Delta \bar{g}^{I}-w^{I} s^{I} \Delta p^{I} / p^{I}\right)=-\sum_{I} \delta p^{I}\left\{w^{I}\left(\mu-\Delta s^{I} \Delta p^{I}\right) / p^{I}+\bar{g}^{I}\right\}
$$

which constitutes a linear system of equations, apart from the quadratic term in the right-hand side, that complements the system of equations associated with the linearization of (4.1):

$$
-\Delta \delta \mathcal{G}^{o}[\boldsymbol{u}, \Delta \boldsymbol{u}]+\sum_{I}\left(\Delta \delta \bar{g}^{I} p^{I}+\delta \bar{g}^{I} \Delta p^{I}\right)=\delta \mathcal{G}^{o}[\boldsymbol{u}]-\sum_{I} \delta \bar{g}^{I} p^{I} .
$$

Here, $\delta \mathcal{G}^{o}[\boldsymbol{u}]$ symbolically denotes the classical weak form of the bulk problem without contact and $\boldsymbol{u}$ symbolically denotes the configurations of the bodies, e.g. in the first Newton-Raphson iteration of the first time/load step $\boldsymbol{u}$ represents the vector of control point positions associated with the reference configuration. The solution of the coupled system (4.6) and (4.7) delivers the update to $\boldsymbol{u}$ as well as to the contact variables $p^{I}$ and, through (4.4), $s^{I}$. The solution strategy will be discussed in the next section.

Before proceeding, it is remarked that the contact contributions to the tangent matrix are standard and require no special discussion: $\left\{\delta \bar{g}^{I}, \Delta \bar{g}^{I}, \Delta \delta \bar{g}^{I}\right\}$ are classical terms and all other terms are straightforward. Clearly, the contributions are symmetric. It is also worth comparing the framework to the AL approach $[1,41,43,59,61]$ as the primal-dual extension based on the functional (2.8). Therein, the weak form (4.7) of the linear momentum balance remains identical. However, the pressure is defined through an augmented Lagrange multiplier $\lambda^{I}$ :

$$
p^{I}=\lambda^{I}+\epsilon \bar{g}^{I} / w^{I} \longrightarrow \Delta p^{I}=\Delta \lambda^{I}+\epsilon \Delta \bar{g}^{I} / w^{I} .
$$

The weak statement (4.6) is replaced by

$$
\sum_{I \in \mathcal{A}} \delta \lambda^{I} \Delta \bar{g}^{I}-\sum_{I \notin \mathcal{A}} \delta \lambda^{I} w^{I} \Delta \lambda^{I} / \epsilon=-\sum_{I \in \mathcal{A}} \delta \lambda^{I} \bar{g}^{I}+\sum_{I \notin \mathcal{A}} \delta \lambda^{I} w^{I} \lambda^{I} / \epsilon .
$$


Note that it is necessary to define an active set $\mathcal{A}: I \in \mathcal{A}$ if $p^{I} \geqslant 0$. The inactive set contribution serves to reset the deactivated multipliers to zero. In view of the considerable algorithmic similarity of the two approaches, the present IP formulation can easily be pursued starting from an AL implementation. Moreover, it is straightforward to switch from the IP formulation to the AL formulation at any stage of the iterative solution scheme by transferring the Lagrange multipliers, although an advantage of such an approach has not been observed for the set of examples investigated in this work.

\subsection{Predictor stage}

At a given load step, the nonlinear system of equations describing the IP method is iteratively solved within a Newton-Raphson scheme. Each iteration step involves two stages within which (4.7) is preserved and (4.6) is successively modified. For further theoretical and practical details on the method chosen, the reader is referred to [15] (Section 14.2 and Chapter 19).

The first predictor (or, affine-scaling) stage starts from the last iterative solution $\left\{\boldsymbol{u}, s^{I}, p^{I}\right\}$ of the NewtonRaphson procedure. To determine the predictor updates, one sets both $\mu$ as well as the quadratic term within the right-hand of side (4.6) to zero. Therefore, the predictor form of (4.6) becomes

$$
\mathcal{R}=-\sum_{I} \delta p^{I} \bar{g}^{I} \longrightarrow \sum_{I} \delta p^{I}\left(\Delta \bar{g}_{p}^{I}-w^{I} s^{I} \Delta p_{p}^{I} / p^{I}\right)=\mathcal{R}
$$

and together with (4.7) delivers the incremental predictor updates $\Delta \boldsymbol{u}_{p}$ and $\Delta p_{p}^{I}$. These are used to determine the predictor update to the slack variables through (4.4) where $\mu$ and the quadratic term are still omitted:

$$
\Delta s_{p}^{I}=-\left(s^{I} p^{I}+s^{I} \Delta p_{p}^{I}\right) / p^{I} .
$$

The computational cost of this first stage is identical to a single iteration based on the AL method - see Eqs. (4.8) and (4.9). The second stage will therefore lead to additional cost. However, the iterative solution $\left\{\boldsymbol{u}, s^{I}, p^{I}\right\}$ will not be updated until the end of the second stage. Consequently, the second stage will make use of the same tangent matrix emanating from (4.7) and (4.6), as well as the same right-hand side from (4.7). New terms will only be added to the right-hand side $\mathcal{R}$ from (4.10), but without the need for additional element-level computation. Consequently, the overall additional cost of the second stage will be due to an extra linear system solver call. Typically this cost can be minimized since, for instance, the factorized tangent matrix can be retained for reuse in the second stage.

\subsection{Corrector stage}

The second corrector stage (in its present form including the so-called centering contribution [15]) starts by setting the barrier parameter $\mu$ via a heuristically chosen centering parameter $\beta$

$$
\beta=\left(\frac{\mu_{p}}{\mu_{o}}\right)^{3} \longrightarrow \mu=\beta \mu_{o} .
$$

Here, the deviation from the complementarity condition in (4.2) is assessed by

$$
\sum_{I} w^{I} \mu_{o}=\sum_{I} w^{I} p^{I} s^{I} \longrightarrow \mu_{o}=\sum_{I} w^{I} p^{I} s^{I} / \sum_{I} w^{I}
$$

for the last iteration, and similarly by

$$
\mu_{p}=\sum_{I} w^{I}\left(p^{I}+\alpha_{p}^{D} \Delta p_{p}^{I}\right)\left(s^{I}+\alpha_{p}^{P} \Delta s_{p}^{I}\right) / \sum_{I} w^{I}
$$

for the predictor update, where the primal and dual predictor scaling factors $\left\{\alpha_{p}^{P}, \alpha_{p}^{D}\right\}$ lie in $(0,1]$.

The right-hand side $\mathcal{R}$ from (4.10) is now updated by adding the previously omitted terms:

$$
\sum_{I} \delta p^{I}\left(\Delta \bar{g}_{c}^{I}-w^{I} s^{I} \Delta p_{c}^{I} / p^{I}\right)=\mathcal{R}-\sum_{I} \delta p^{I} w^{I}\left(\mu-\Delta s_{p}^{I} \Delta p_{p}^{I}\right) / p^{I}
$$


Together with (4.7), this updated system of equations delivers the incremental corrector updates $\Delta \boldsymbol{u}_{c}$ and $\Delta p_{c}^{I}$ which, through (4.4), determine the corrector update to the slack variables:

$$
\Delta s_{c}^{I}=\left(\mu-s^{I} p^{I}-s^{I} \Delta p_{c}^{I}-\Delta s_{p}^{I} \Delta p_{p}^{I}\right) / p^{I} .
$$

Using corrector scaling factors $\left\{\alpha_{c}^{P}, \alpha_{c}^{D}\right\}$ in $(0,1]$, the final algorithmic update to the solution is

$$
\boldsymbol{u}=\boldsymbol{u}+\Delta \boldsymbol{u}_{c}, \quad s^{I}=s^{I}+\alpha_{c}^{P} \Delta s_{c}^{I}, \quad p^{I}=p^{I}+\alpha_{c}^{D} \Delta p_{c}^{I}
$$

followed by a new two-stage Newton-Raphson iteration until convergence within a load step.

The step size choices are made such that $s^{I}$ and $p^{I}$ remain positive. This is achieved through the fraction to the boundary rules

$$
\alpha_{c}^{P}=\min \left\{1, \min _{I: \Delta s_{c}^{I}<0}-\frac{\gamma s^{I}}{\Delta s_{c}^{I}}\right\}, \quad \alpha_{c}^{D}=\min \left\{1, \min _{I: \Delta p_{c}^{I}<0}-\frac{\gamma p^{I}}{\Delta p_{c}^{I}}\right\} .
$$

The predictor scaling factors $\left\{\alpha_{p}^{P}, \alpha_{p}^{D}\right\}$ in (4.14) are defined similarly. The constant $\gamma$, typically $[0.9,1)$, determines how close to zero the slack variables and the Lagrange multipliers are allowed to approach. The choices regarding the barrier parameter and solution update are not unique and may be altered to improve performance. For instance, one may set minimum and maximum values for $\beta$ or the update to $\boldsymbol{u}$ may also be scaled, e.g. by $\alpha_{c}^{P}$. Also, the scheme above drives $\mu$ to zero aggressively. Modifications can slow down the rate at which this is carried out or a cutoff may be employed.

\subsection{Efficiency and robustness}

A summary of the primal-dual solution algorithm is presented in Table 1. Unlike the AL approach, the primal-dual algorithm is not expected to converge quadratically due to the continuous update of the barrier parameter $\mu$. Nevertheless, the algorithm converges superlinearly, in fact very close to quadratically. Moreover, due to the lack of an active set, the overall efficiency of the method can be superior to the AL method despite the fact that the time per iteration is higher due to one extra linear system solve. From a robustness point of view, on the other hand, it was observed that the IP method is just as robust as the AL method when multiple time or load steps are not required. Otherwise, the IP method fails due to the rapid reduction of the barrier parameter across the steps. However, the predictor-corrector scheme presented here is not unique and modifications can alter the degree of robustness $[15,16]$. See Section 5 for further discussion.

\subsection{Primal algorithm}

The primal strategy outlined in Section 3.3 explicitly enforces the complementarity condition via (3.10). The resulting algorithm does not require a two-stage iteration and operates through a constant barrier parameter $\mu$, as summarized in Table 2. Here, the non-smoothness introduced by the scaling factor has similar consequences as the non-smoothness introduced by the active set of the primal EP method. Therefore, the primal strategy delivers asymptotically quadratic convergence since the scaling factor consistently takes the value 1 near convergence.

An alternative primal formulation may be obtained by modifying the primal-dual algorithm in a straightforward fashion. For this purpose, one omits the quadratic term in the linearization (4.3) of the complementarity condition and, hence, in the update (4.4) to $s^{I}$. In other words, one simply carries out a standard linearization of the system of equations. Consequently, as in the primal-dual case, both the gap and the complementarity conditions are satisfied only at convergence. The resulting algorithm, still operating through a constant $\mu$, also converges asymptotically quadratically and is summarized in Table 3. With some abuse of terminology, this version is also referred to as primal since it converges to the same result as the previous version. The additional cost due to the introduction of the additional degrees of freedom $p^{I}$ is not significant. In view of the observations to be stated in Section 5.2, this will be the preferred primal IP algorithm.

In either case, the barrier parameter needs to be chosen sufficiently small to ensure a physically reasonable solution. In the primal EP method, and in particular for two highly deformable bodies in large sliding contact, 
Table 1

Algorithm for the mortar-based primal-dual IP method.

1. Initialize. Within a load step, the solution $\left\{\boldsymbol{u}, s^{I}, p^{I}\right\}$ (hence $\bar{g}^{I}=\left\langle N^{I} g\right\rangle$ ) from the last Newton-Raphson iteration is known. The weights $w^{I}=\left\langle N^{I}\right\rangle$ are iteration independent. (For the first load step set, e.g., $s^{I}=\left|\bar{g}^{I}\right| / w^{I}$ and $p^{I}=\mu_{o} / s^{I}$ with a chosen $\mu_{o}$.)

2. Linearized System Setup. The two-stage Newton-Raphson iteration requires the solution of (4.7)

(1) $-\Delta \delta \mathcal{G}^{o}[\boldsymbol{u}, \Delta \boldsymbol{u}]+\sum_{I}\left(\Delta \delta \bar{g}^{I} p^{I}+\delta \bar{g}^{I} \Delta p^{I}\right)=\delta \mathcal{G}^{o}[\boldsymbol{u}]-\sum_{I} \delta \bar{g}^{I} p^{I}$,

together with (4.6) for a prescribed right-hand side $\mathcal{R}$ :

(2) $\sum_{I} \delta p^{I}\left(\Delta \bar{g}^{I}-w^{I} s^{I} \Delta p^{I} / p^{I}\right)=\mathcal{R}$

Compute the stage-independent tangent matrices emanating from the left-hand sides of (1) and (2), as well as the stage-independent right-hand side of (1).

3. Predictor Stage. Determine the predictor updates $\Delta \boldsymbol{u}_{p}$ and $\Delta p_{p}^{I}$ by solving (1) and (2) using

$\mathcal{R}=-\sum_{I} \delta p^{I} \bar{g}^{I}$

The slack variable updates follow from the predictor form (4.11) of (4.4):

$\Delta s_{p}^{I}=-\left(s^{I} p^{I}+s^{I} \Delta p_{p}^{I}\right) / p^{I}$

4. Barrier Parameter Update. Calculate predictor scaling factors using, e.g., $\gamma \in[0.9,1)$

$\alpha_{p}^{P}=\min \left\{1, \min _{I: \Delta s_{p}^{I}<0}-\gamma s^{I} / \Delta s_{p}^{I}\right\}, \quad \alpha_{p}^{D}=\min \left\{1, \min _{I: \Delta p_{p}^{I}<0}-\gamma p^{I} / \Delta p_{p}^{I}\right\}$

and base quantities

$\mu_{o}=\sum_{I} w^{I} p^{I} s^{I} / \sum_{I} w^{I}, \quad \mu_{p}=\sum_{I} w^{I}\left(p^{I}+\alpha_{p}^{D} \Delta p_{p}^{I}\right)\left(s^{I}+\alpha_{p}^{P} \Delta s_{p}^{I}\right) / \sum_{I} w^{I}$

to set the barrier parameter

$\beta=\left(\mu_{p} / \mu_{o}\right)^{3} \longrightarrow \mu=\beta \mu_{o}$

5. Corrector Stage. Determine the corrector updates $\Delta \boldsymbol{u}_{c}$ and $\Delta p_{c}^{I}$ by solving (1) and (2) after the update

$\mathcal{R}=\mathcal{R}-\sum_{I} \delta p^{I} w^{I}\left(\mu-\Delta s_{p}^{I} \Delta p_{p}^{I}\right) / p^{I}$.

The slack variable updates follow from the corrector form (4.16) of (4.4):

$\Delta s_{c}^{I}=\left(\mu-s^{I} p^{I}-s^{I} \Delta p_{c}^{I}-\Delta s_{p}^{I} \Delta p_{p}^{I}\right) / p^{I}$.

6. Iterative Solution Update. Compute corrector scaling factors

$\alpha_{c}^{P}=\min \left\{1, \min _{I: \Delta s_{c}^{I}<0}-\gamma s^{I} / \Delta s_{c}^{I}\right\}, \quad \alpha_{c}^{D}=\min \left\{1, \min _{I: \Delta p_{c}^{I}<0}-\gamma p^{I} / \Delta p_{c}^{I}\right\}$

and update the solution based on the corrector stage solution:

$\boldsymbol{u}=\boldsymbol{u}+\Delta \boldsymbol{u}_{c}, \quad s^{I}=s^{I}+\alpha_{c}^{P} \Delta s_{c}^{I}, \quad p^{I}=p^{I}+\alpha_{c}^{D} \Delta p_{c}^{I}$.

7. Check convergence. Compute the iteration error and reiterate if necessary.

it is well-known that the solution often displays the need for a larger $\epsilon$ yet convergence cannot be achieved when it is increased or the resulting robustness is significantly inferior compared to the AL method. Contrary to expectation, it was observed that this problem is not nearly as significant in the primal IP method $-\mu$ can be chosen satisfactorily small. In fact, it was observed that (i) the algorithm challenges the AL approach in all the cases tested, even displaying better robustness at the same cost, and (ii) it is more robust than the predictor-corrector scheme adopted in this work. With respect to the latter observation, it should be remarked that the construction of robust primal-dual approaches is an active research area for the IP method where proper choices of the centering parameter and the fraction to the boundary rules directly impact efficiency and 
Table 2

Algorithm for the mortar-based primal IP method: Version 1.

1. Initialize. Within a load step, the solution $\left\{\boldsymbol{u}, s^{I}\right\}$ (hence $\bar{g}^{I}=\left\langle N^{I} g\right\rangle$ ) from the last Newton-Raphson iteration is known. Constants $w^{I}=\left\langle N^{I}\right\rangle$ are iteration independent. (For the first load step set, e.g., $s^{I}=\left|\bar{g}^{I}\right| / w^{I}$.)

2. Primal Formulation. The barrier parameter $\mu$ is prescribed to a fixed value, hence $p^{I}=\mu / s^{I}$ is known. Using $\Delta s^{I}$ from the linearized gap condition (4.3), obtain the pressure linearization:

$$
\Delta p^{I}=\Delta\left(\mu / s^{I}\right)=-p^{I} \Delta s^{I} / s^{I}=\left(p^{I} s^{I}+p^{I} \bar{g}^{I} / w^{I}+p^{I} \Delta \bar{g}^{I} / w^{I}\right) / s^{I} .
$$

3. Linearized System Solution. The Newton-Raphson iteration update $\Delta \boldsymbol{u}$ follows from (4.7)

$$
-\Delta \delta \mathcal{G}^{o}[\boldsymbol{u}, \Delta \boldsymbol{u}]+\sum_{I}\left(\Delta \delta \bar{g}^{I} p^{I}+\delta \bar{g}^{I} \Delta \bar{g}^{I} p^{I} / w^{I} s^{I}\right)=\delta \mathcal{G}^{o}[\boldsymbol{u}]-\sum_{I} \delta \bar{g}^{I}\left(2 p^{I}+p^{I} \bar{g}^{I} / w^{I} s^{I}\right),
$$

which delivers $\Delta \bar{g}^{I}$ and hence $\Delta s^{I}$.

4. Iterative Solution Update. Compute a scaling factor using, e.g., $\gamma \in[0.9,1)$

$$
\alpha=\min \left\{1, \min _{I: \Delta s^{I}<0}-\gamma s^{I} / \Delta s^{I}\right\}
$$

and update the solution based on the corrected increments:

$\boldsymbol{u}=\boldsymbol{u}+\Delta \boldsymbol{u}, \quad s^{I}=s^{I}+\alpha \Delta s^{I} \longrightarrow p^{I}=\mu / s^{I}$.

5. Check convergence. Compute the iteration error and reiterate if necessary.

robustness. It is believed that the adoption of alternative strategies that deliver superior performance can be pursued based on the present framework.

\section{Numerical investigations}

In this section, the observations summarized in the previous sections as well as additional aspects of the interior point method are demonstrated by numerical examples. Where possible, a compact presentation is pursued by borrowing test cases from earlier works where detailed descriptions of the loading scenarios may be found. Some relatively straightforward remarks are not explicitly discussed. For instance, the enforcement of the contact constraints at the integration points leads to surface locking (Section 3.1) and the patch test is passed to high accuracy (Section 3.2). These would follow in a similar fashion as in earlier isogeometric contact studies of surface locking and patch tests based on penalty approaches [53]. In all examples, $\gamma=0.95$ has been employed in the IP algorithms summarized in Tables 1 and 3 . The value of the barrier parameter will be noted.

\subsection{Hertzian contact}

The classical Hertzian contact is a convenient setting since it is a materially and geometrically linear problem that helps assess the performance of the optimization algorithm alone. For instance, the stiffness matrix needs to be computed only once so that comparisons of simulation times are indicative of the algorithm efficiency. For this purpose, the plane-strain setting with two bodies is considered, based on the order elevation and knot refinement schemes discussed for this problem in [25]. The Young's modulus is $E=1$ and the Poisson's ratio is $v=0.3$. Coarser and finer discretizations respectively halve and double the angular NURBS discretization of the default case where the slave is assigned 96 elements and the master 48 . The discretizations in the radial direction are the same, fixed and based on 48 linear Lagrange elements. The default NURBS order is two $\left(\mathcal{N}^{2}\right)$.

Fig. 1 summarizes fundamental aspects of the IP algorithm. For the primal IP method (Table 3), the choice of the barrier parameter $\mu$ directly affects the quality of the solution, which quickly approaches the exact solution as $\mu$ is decreased. The primal-dual IP method (Table 1) eliminates the arbitrariness due to the choice of $\mu$. The solution obtained with an initial guess $\mu_{o}=10^{-5}$ overlaps with the AL one (see Eq. (4.9)) since both methods enforce the contact constraints (2.4) exactly. In order to remove the observed oscillations, the discretization must be refined by knot insertion. The mortar-based contact algorithm is uniformly applicable to all 
Table 3

Algorithm for the mortar-based primal IP method: Version 2.

1. Initialize. Within a load step, the solution $\left\{\boldsymbol{u}, s^{I}, p^{I}\right\}$ (hence $\bar{g}^{I}=\left\langle N^{I} g\right\rangle$ ) from the last Newton-Raphson iteration is known. The weights $w^{I}=\left\langle N^{I}\right\rangle$ are iteration independent. (For the first load step set, e.g., $s^{I}=\left|\bar{g}^{I}\right| / w^{I}$ and $p^{I}=\mu / s^{I}$ where $\mu$ is prescribed to a constant value.)

2. Linearized System Solution. The Newton-Raphson iteration requires the solution of (4.7)

$-\Delta \delta \mathcal{G}^{o}[\boldsymbol{u}, \Delta \boldsymbol{u}]+\sum_{I}\left(\Delta \delta \bar{g}^{I} p^{I}+\delta \bar{g}^{I} \Delta p^{I}\right)=\delta \mathcal{G}^{o}[\boldsymbol{u}]-\sum_{I} \delta \bar{g}^{I} p^{I}$,

together with (4.6) by omitting the quadratic terms:

$\sum_{I} \delta p^{I}\left(\Delta \bar{g}^{I}-w^{I} s^{I} \Delta p^{I} / p^{I}\right)=-\sum_{I} \delta p^{I}\left(w^{I} \mu / p^{I}+\bar{g}^{I}\right)$.

The solution delivers the update $\Delta \boldsymbol{u}$ and hence $\Delta s^{I}$, using (4.4) without the quadratic terms.

3. Iterative Solution Update. Compute scaling factors using, e.g., $\gamma \in[0.9,1)$

$\alpha^{P}=\min \left\{1, \min _{I: \Delta s^{I}<0}-\gamma s^{I} / \Delta s^{I}\right\}, \quad \alpha^{D}=\min \left\{1, \min _{I: \Delta p^{I}<0}-\gamma p^{I} / \Delta p^{I}\right\}$

and update the solution based on the corrected increments:

$\boldsymbol{u}=\boldsymbol{u}+\Delta \boldsymbol{u}, \quad s^{I}=s^{I}+\alpha^{P} \Delta s^{I}, \quad p^{I}=p^{I}+\alpha^{D} \Delta p^{I}$.

4. Check convergence. Compute the iteration error and reiterate if necessary.

orders of NURBS discretizations, although the solution remains relatively unaffected by order elevation at a fixed resolution. A preliminary analysis additionally indicated that the order of convergence does not improve with order elevation. Discussions of optimal convergence for mortar-based higher-order NURBS and Lagrangian discretizations may be found in $[52,50,55]$. Presently, the practical advantage of order elevation appears when there is large sliding as will be observed in Section 5.4.

A comparison of the primal-dual IP and the AL methods is provided in Fig. 2 for the test cases of Fig. 1. Here, the iteration error is monitored to convergence. Both methods are relatively insensitive to order elevation. However, a clear performance advantage is observed for the IP method: convergence is faster, asymptotically near-quadratic, smooth and monotonic. The smooth monotonic convergence of the IP method is due to the absence of an active set. Indeed, the AL method delivers quadratic convergence only after the active set settles. The advantage becomes clearer for knot refinement. With an increase in the number of contact degrees of freedom, both methods require a larger number of iterations to convergence. However, the AL method requires almost twice the number of iterations in each case. Since the primal-dual IP method requires two solves per iteration, the overall solution times do not show the same ratio although the IP method still displays a clear advantage. It is noted that each solve per IP solution step was carried out independently, without taking advantage of the fact that the tangent matrix remains identical. Therefore, the solution times for the IP method leave room for improvement. It is also noted that for the application of the AL method, the penalty parameter is chosen as $\epsilon=10$. At this value, a pure penalty approach would deliver a solution that is similar to the primal IP solution with $\mu=10^{-5}$. The AL method performance is insensitive to the choice of $\epsilon$, although larger choices start to display typical ill-conditioning problems. The primal-dual IP method performance is slightly sensitive to the initial choice $\mu_{o}$ since it must be driven to zero with iterations. However, the increase in the number of iterations is small (roughly one additional iteration for one order of magnitude increase in $\mu_{o}$ ). Similar to the AL method, smaller choices of $\mu_{o}$ render the primal-dual IP method prone to illconditioning.

\subsection{Finite Hertzian-type contact}

A finite Hertzian-type contact problem is considered, based on the material, geometry and loading choices in [56] with the extension of a deformable master body. The goal is to assess the effect of significant nonlinearity in the problem. Here, two bodies with a Neo-Hooke type elastic response $(E=10, v=0.3)$ are pressed against each other under displacement control of the top surface of the upper slave body. At the coarse 


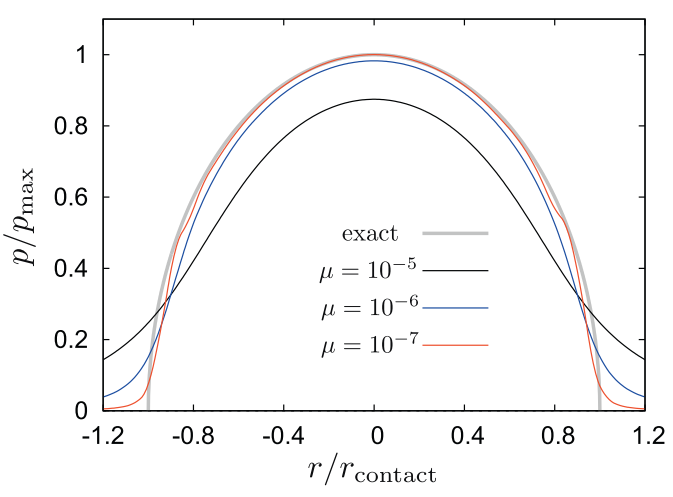

(a) effect of the barrier parameter

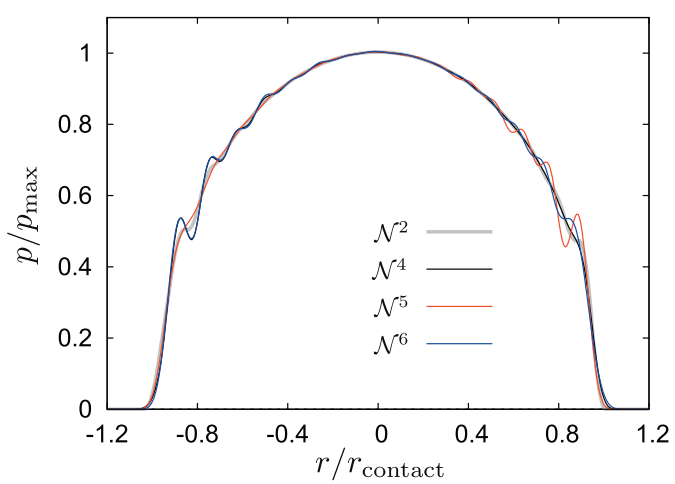

(c) order elevation

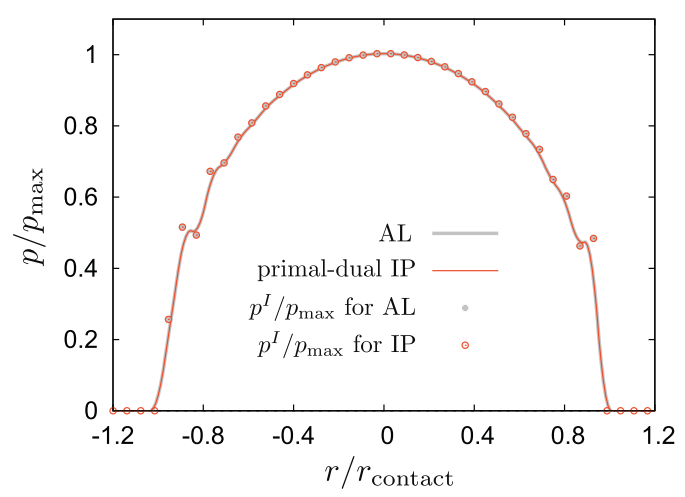

(b) comparison with $\mathrm{AL}$

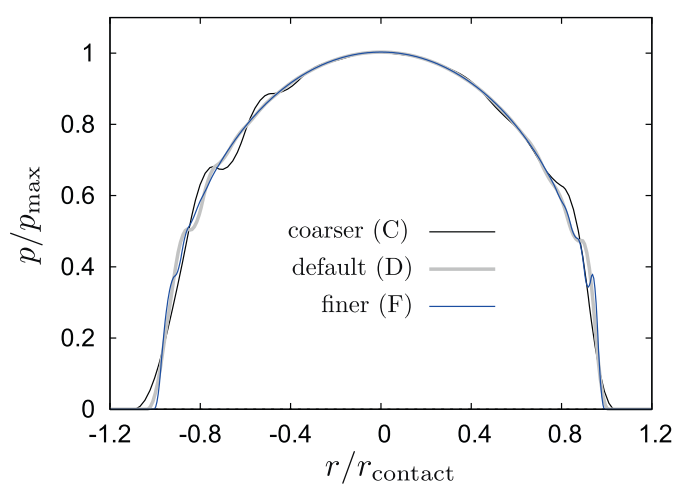

(d) knot insertion

Fig. 1. The Hertzian contact problem of Section 5.1: (a) the barrier parameter is varied using the primal IP method (version 2), (b) the primal-dual IP method is compared with the AL method, (c) the effect of order elevation as well as (d) knot refinement is monitored. $p_{\text {max }}$ is the maximum pressure and $\mathrm{r}$ is the distance from the center of the contact zone of width $2 r_{\text {contact }}$. After order elevation followed by knot refinement on the original geometry, the interface control points are concentrated in the vicinity of the contact zone by relocating the knots [25]. The procedure does not guarantee a symmetric mesh, which is the source of the unsymmetric pressure distribution. For the portion of the mesh within the contact zone, the unsymmetry in the mesh is observable from the unsymmetric positions of the control points near $r / r_{\text {contact }}= \pm 0.8$ in (b).

discretization, the slave is discretized with $6 / 3 \mathcal{N}^{2}$ elements in the horizontal/vertical directions and the master with 4/2. From the coarse (C) to the medium (M) and from the medium to the fine (F) discretization, the number of elements in each direction is doubled. At the finest resolution, there are more than 12,000 control points.

Fig. 3 displays the problem setting based on a relatively large barrier parameter such that the primal IP method (Table 3) delivers a significant gap in comparison with the primal-dual one. The primal-dual IP and AL methods are compared in Fig. 4(a). Clearly, in comparison with the linear setting of Section 5.1, the primal-dual IP method appears to lose its advantage in terms of efficiency, even requiring a larger number of iterations at the finest resolution despite displaying initial faster convergence. In terms of computation time, the primal-dual IP method requires two solves per iteration so that the overall computation time is also higher. However, the method is just as robust as the AL approach for this problem. In particular, the significant deformation visible in Fig. 3 has been applied in one step at all resolutions.

In order to highlight an advantage of the IP method, the EP penalty approach is compared with the primal IP method. Either one is inferior to the methods discussed above so that the loading must be applied in two steps to ensure convergence. Even then, the penalty method fails to converge for values of $\epsilon$ above 10 which, however, delivers an unacceptable solution quality with excessive penetration and little deformation of the bodies. The primal IP method, on the other hand, already delivers an acceptable solution at $\mu=10^{-2}$. Beyond $\mu=10^{-3}$ the solution is indistinguishable from the primal-dual one although $\mu$ can be decreased even further 


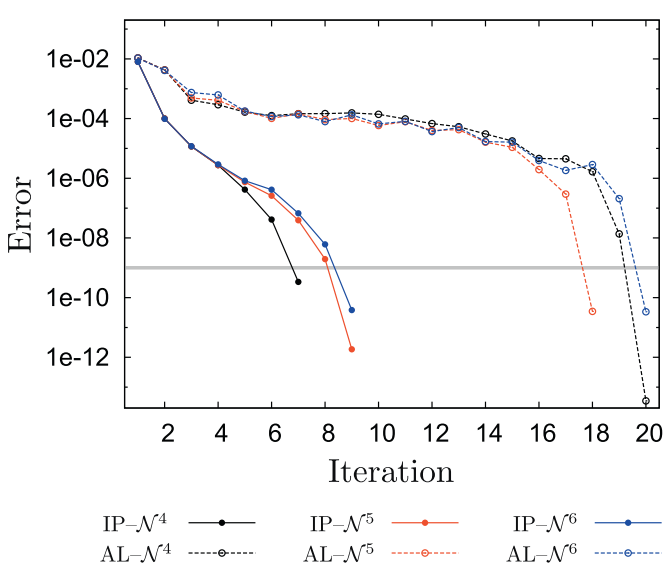

(a) order elevation

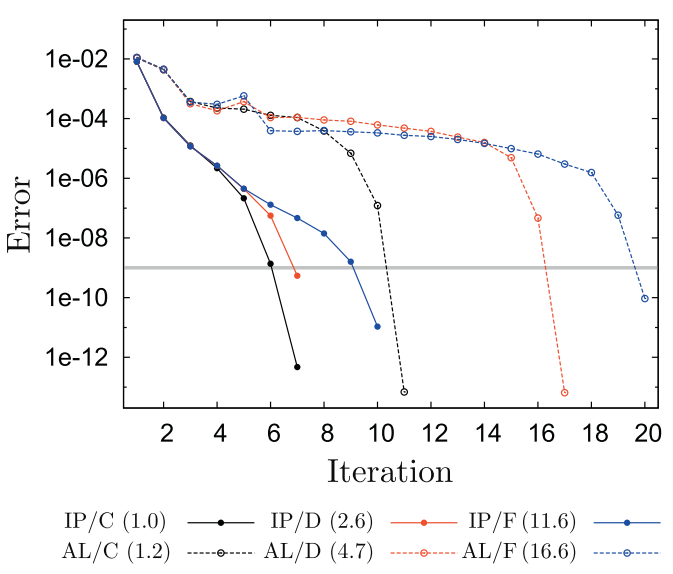

(b) knot refinement

Fig. 2. The convergence behaviors of the AL and the primal-dual IP method are summarized for cases (c) and (d) in Fig. 1. The error is defined as $\|\Delta \boldsymbol{u}\|_{2} /\left\|\boldsymbol{u}_{\text {old }}\right\|_{2}$. For knot refinement based on coarse (C), default (D) and fine (F) $\mathcal{N}^{2}$ discretizations, the numbers in parentheses denote the relative simulation times (based on the average of 100 (C), 10 (D) and 5 (F) runs), with the coarse IP solution as the reference. The gray line indicates the convergence tolerance $\left(10^{-9}\right)$.

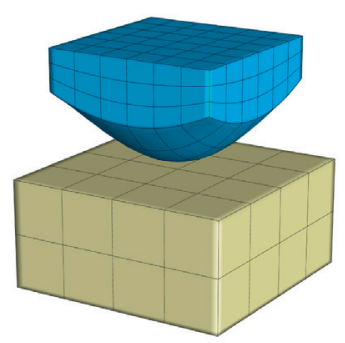

(a) undeformed

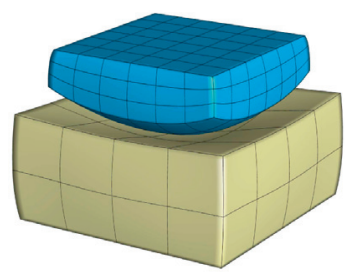

(b) deformed

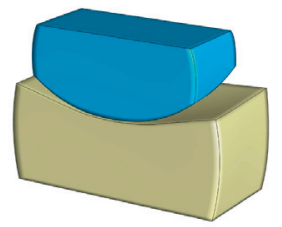

(c) positive gap

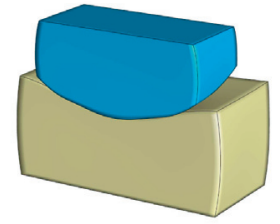

(d) primal-dual IP

Fig. 3. The finite Hertzian-type contact problem is depicted at the coarse resolution: (a) to (c) are based on the primal IP method (version 2) with $\mu=10^{-1}$, which results in a visible gap. The primal-dual IP method also employs this value as the starting point $\mu_{o}$ but drives the gap to zero.

by several orders of magnitude. The convergence behavior as $\mu$ is adjusted is monitored in Fig. 4(b) for the second load step. In general, the choice of the barrier parameter in the primal IP method should be made judiciously by taking into account the stiffness of the bodies in contact. Similar to the EP penalty approach, the number of iterations to convergence increases as the contact gap is driven to zero by adjusting the regularization parameter. At an acceptable quality, the primal IP method is still more expensive than the AL method since the IP formulation requires integrating over all the contact surface versus only the active ones. In view of the fact that the loading is typically applied at multiple steps, it is interesting to observe that the primal IP method can challenge the primal-dual one for nonlinear problems in terms of efficiency and also display a close level of robustness.

By construction, both versions of the primal IP method deliver the same result although the iterative convergence behavior of each version is different as summarized in Fig. 4(c). Version 1 of the algorithm fails to converge in 2 steps for the chosen values of $\mu$. Only $\mu=10^{-3}$ converges with 3 steps. With 4 steps or larger, version 1 converges for all choices of $\mu$. This indicates that version 2 of the primal algorithm is more robust and it also appears to converge faster. It is recalled that the algorithmic difference between the two versions is the indirect relation between the slack variable and the pressure as well as their independently scaled updates in version 2 (see Table 3, step 3). In view of the observed advantages of version 2, version 1 will not be investigated further in the remaining examples. 


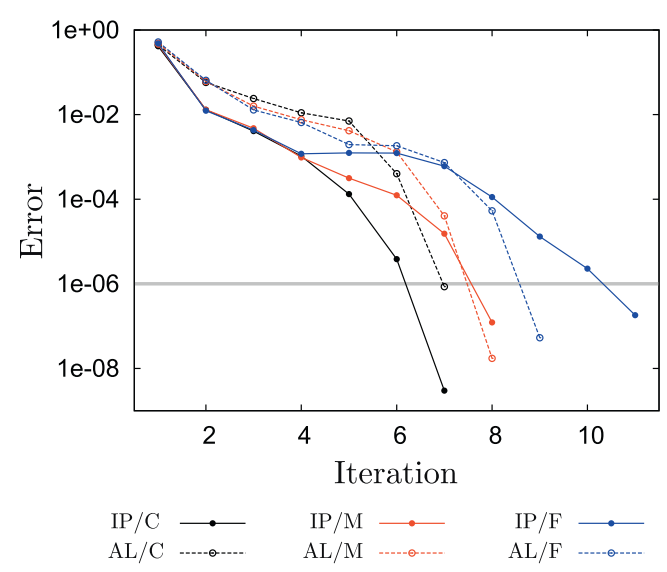

(a) knot refinement

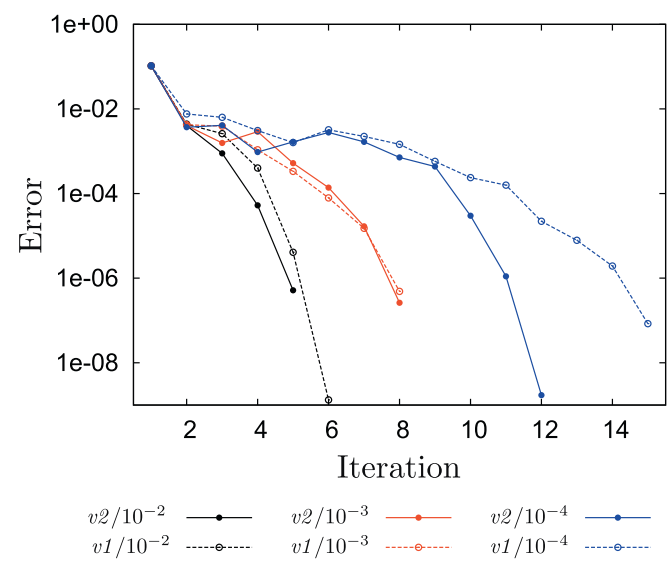

(c) primal algorithm: versions 1 and 2

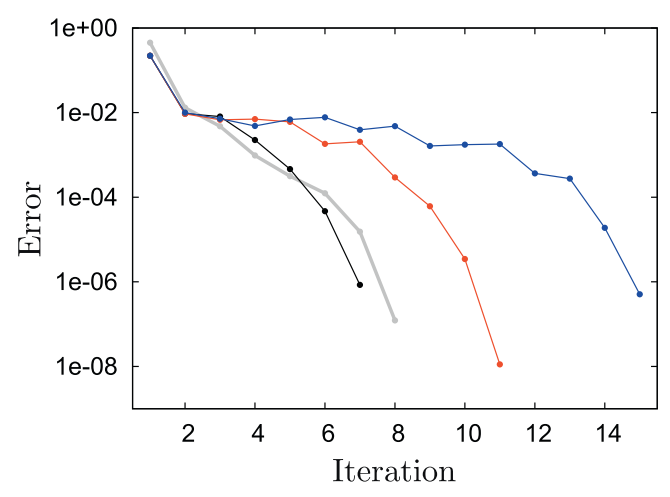

$\begin{aligned} \text { primal-dual IP } \longrightarrow & \mu=10^{-3} \longrightarrow \\ \mu=10^{-2} \longrightarrow & \mu=10^{-4} \longrightarrow\end{aligned}$

(b) barrier adjustment at resolution $\mathrm{M}$

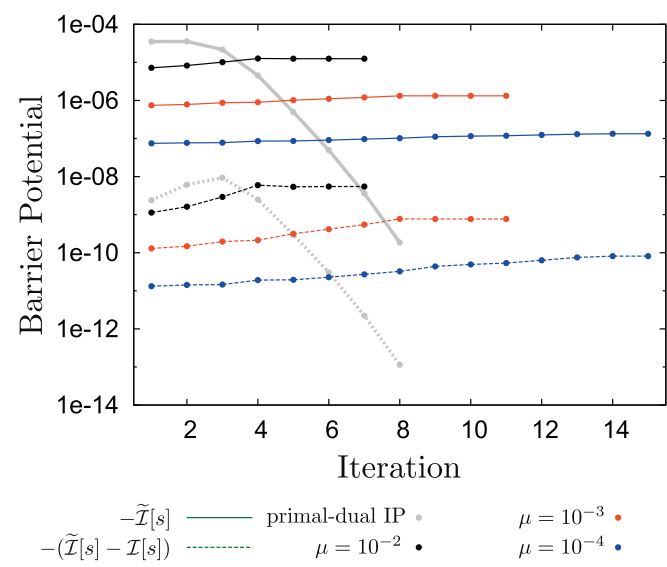

(d) barrier potential comparison

Fig. 4. For the problem of Section 5.2, the convergence behaviors of AL and the IP method are summarized. The error is defined as $\|\Delta \boldsymbol{u}\|_{2} /\left\|\boldsymbol{u}_{\text {old }}\right\|_{2}$. Coarse (C), medium (M) and fine (F) $\mathcal{N}^{2}$ discretizations at compared in (a) where the IP method is based on the primaldual approach. The gray line indicates the convergence tolerance $\left(10^{-6}\right)$. In (b), the primal IP method (version 2) with various barrier parameter values is compared with the primal-dual method. In (c), versions $1(v 1)$ and $2(v 2)$ of the primal algorithm are compared at different $\mu$ values for the last step of a 4-step loading. In (d), following (b), the corresponding barrier potentials for the original and approximate formulations are compared in the context of the discussion of the inequality (3.13).

The original barrier potential is compared with the approximate one in Fig. 4(d). As expected, smaller choices of $\mu$ within the primal formulation delivers smaller values for the potentials. It is observed that this also ensures a vanishing gap between the original and approximate potentials. Moreover, since $\mu$ is driven to zero automatically within the primal-dual formulation, both the potentials and their difference are driven to zero with iterations. Similar trends are observed for order elevation and knot refinement, the former having a weaker influence compared to varying $\mu$ while the latter has an effect of comparable magnitude.

\subsection{Thick-walled shells in sliding contact}

In this section, the large sliding contact of two thick-walled shells is considered at the finite deformation regime, exactly following the setup in [56] - see Fig. 5. Contrary to the previous two examples, this problem requires multiple time steps by construction, chosen to be 20 . As time progresses, contact is first initiated and then lost. Three $\mathcal{N}^{2}$ discretizations are employed, with respectively 10/10 (coarse), 20/10 (medium) and 30/20 (fine) elements on the slave/master in the horizontal directions to monitor the normal $\left(F_{N}\right)$ and tangential $\left(F_{T}\right)$ forces acting on the master. The thickness direction had two elements in all cases. 


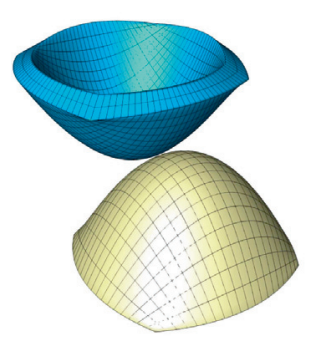

(a) time ratio $=0$

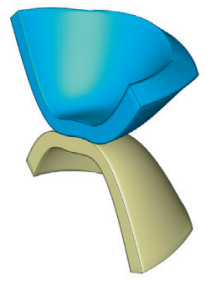

(b) time ratio $=0.4$

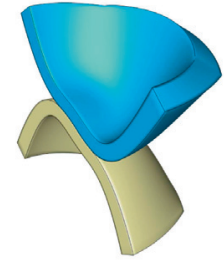

(c) time ratio $=0.6$

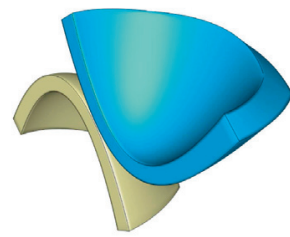

(d) time ratio $=1$

Fig. 5. The problem of Section 5.3 is summarized at the fine $\mathcal{N}^{2}$ discretization based on the AL method. The upper (slave) thick-walled shell is moved over the lower stationary one under displacement control in 20 steps. Time ratio indicates simulation progress.

Due to multiple load steps, the primal-dual IP algorithm in Table 1 usually succeeds in the first few time steps but subsequently drives $\mu$ to too small a value that causes ill-conditioning and hence the failure of the algorithm. A simple modification, such as using a cut-off on $\mu$ or resetting its value to $\mu_{o}$ at each time step (cold-start), does not lead to robustness. Consequently, the primal-dual version of the IP method requires improvement with respect to the update of the barrier parameter through multiple time/load steps, which can be accomplished by adopting warm-start strategies [62,63]. Strategies may also be adopted from primal-dual IP algorithms that have successfully been tailored for similar nonlinear constraint problems arising in elastoplasticity [64]. This is left for a future study. On the other hand, in view of the results of the previous section, the primal IP algorithm in Table 3 is expected to be not only efficient but also as robust as the AL method. Hence, it will be employed in the remaining studies.

Results based on the primal IP method are summarized in Fig. 6. The best EP penalty method solution is obtained at $\epsilon=1$, beyond which the method fails to converge. This value of $\epsilon$ significantly underestimates the solution obtained with the AL method. It should be noted that 20 steps lead to convergence problems for the AL method for all choices of $\epsilon .40$ steps were chosen instead. 20 steps was sufficient for all the IP method applications, indicating that the primal IP method can potentially display higher robustness. $\mu=10^{-3}$ results in an overestimate of the solution. However, the barrier parameter can easily be reduced further by several orders of magnitude. At $\mu=10^{-5}$, the solution is already indistinguishable from the AL one. While the computations were carried out at the medium resolution, the results are observed to be very close to the fine resolution results and the evolution of the forces is smooth throughout the computation, as expected due to the $\mathcal{C}^{1}$-continuity of the $\mathcal{N}^{2}$ discretization and the mortar-based contact algorithm.

\subsection{Ironing with large indentation}

As a last example, the robustness of the primal IP method (Table 3) is compared with the AL method. For this purpose, the classical ironing problem is considered (Fig. 7). It is known that the solution of this problem can be challenging at high stiffness ratios of the two bodies if the harder one is dragged while it significantly indents the softer one [33]. For this analysis, the setup follows [58] in two and three dimensions, with the modification that the stiffness of the upper (slave) body will be increased with respect to the fixed stiffness of the lower one, hence making the indentation more severe. The discretizations of the two bodies are fixed to three $\mathcal{N}^{2}$ elements in the vertical direction and to five for the horizontal direction of the slave. The master body will be discretized with varying orders in the horizontal directions using ten elements in the dragging direction and five in the remaining one for the three-dimensional setup.

Higher-order discretizations are expected to enable the solution of the problem even at coarse dicretizations by allowing the softer body to conform perfectly to the harder surface (Fig. 7). Even though the discretizations are $\mathcal{C}^{1}$-continuous in all cases, the degree of conformity decreases with decreasing order, which reflects as an increasing amplitude of oscillations for the normal $\left(F_{N}\right)$ and, in particular, the tangential $\left(F_{T}\right)$ forces acting on the master. See also $[58,57]$ for studies of the degree of smoothness for isogeometric mortar-based discretizations in the presence of friction. The solution of the problem with 20 normal (indentation) and 200 tangential 

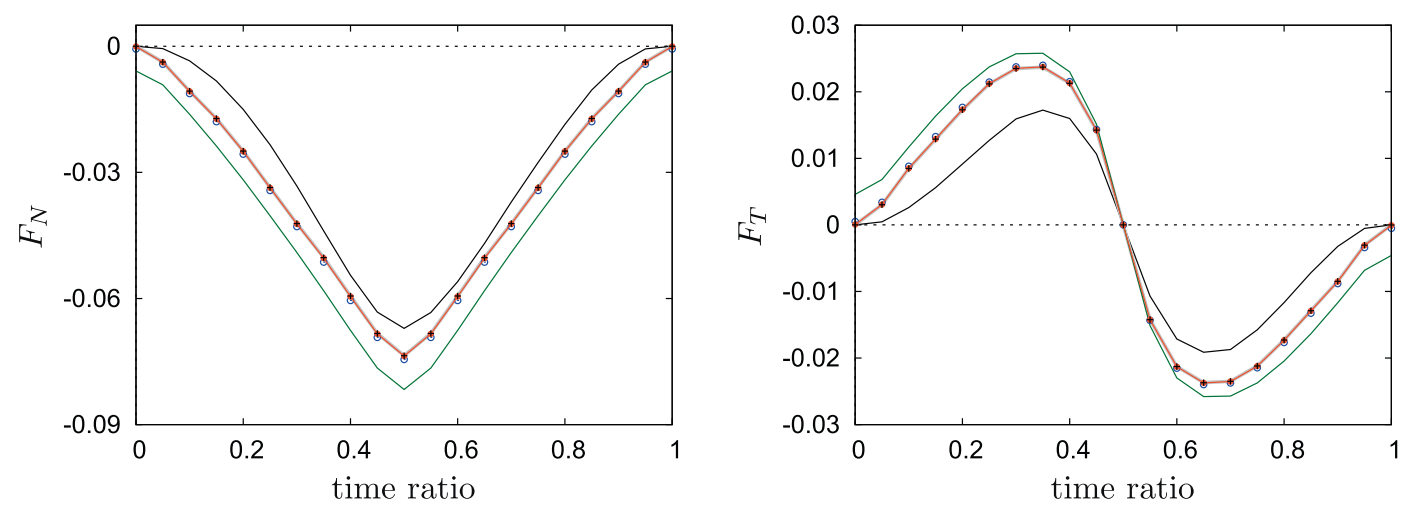

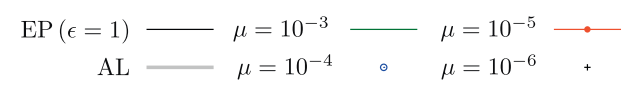

(a) barrier adjustment at medium resolution

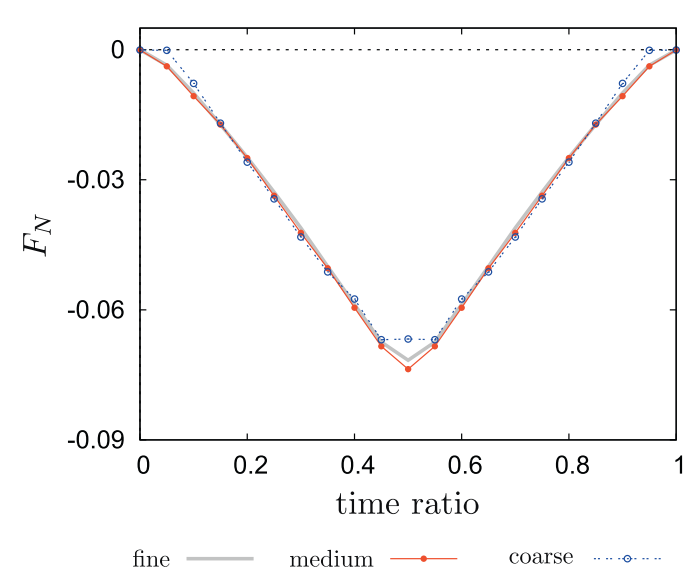

(c) knot refinement with $\mu=10^{-5}$

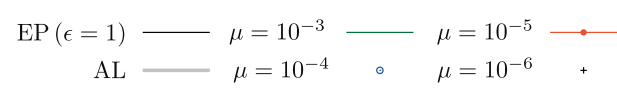

(b) barrier adjustment at medium resolution

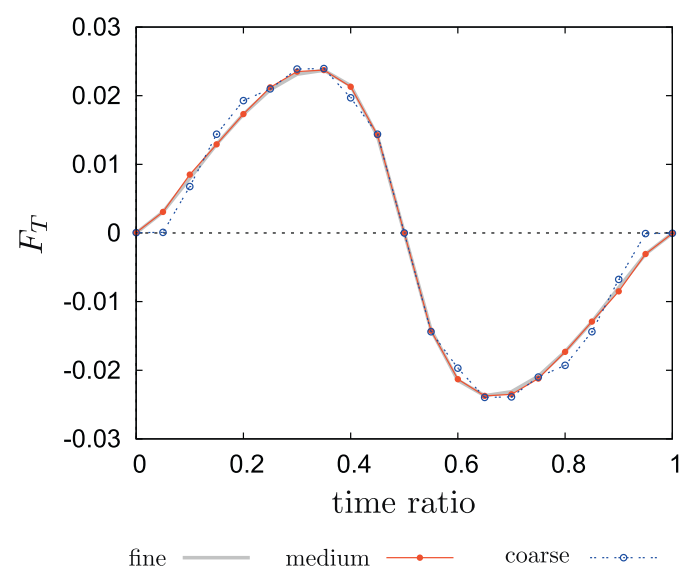

(d) knot refinement with $\mu=10^{-5}$

Fig. 6. Problem of Section 5.3 is analyzed. (a) and (b) Compare the primal IP method (version 2) with the penalty and AL methods at a fixed resolution. (c) and (d) Consider the effect of resolution using the primal IP method with a fixed barrier parameter.

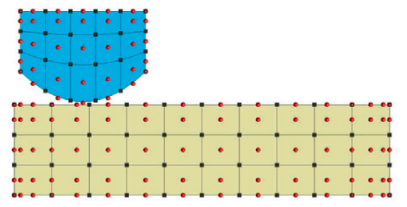

(a) discretization

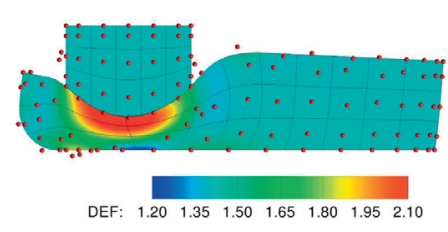

(b) end of indentation

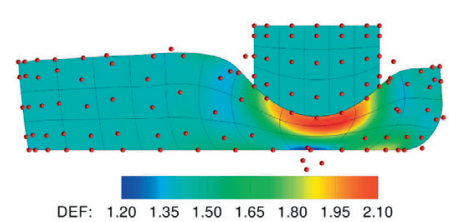

(c) end of dragging

Fig. 7. The ironing problem of Section 5.4 with large indentation is summarized. The instances are obtained from a simulation where the upper (slave) body is $10^{4}$ times stiffer than the lower one. The bodies are discretized with $\mathcal{N}^{2}$ in the vertical direction and $\mathcal{N}^{6}$ in the horizontal one. $D E F$ indicates the magnitude of the deformation gradient tensor.

(dragging) stages based on the AL method reflects these oscillations (Fig. 8(a) and (b)) and how they diminish with order elevation. Orders lower than four did not deliver a reliable performance due to extreme oscillations at this resolution, hence they are omitted from the investigation. As the barrier parameter is decreased by orders of magnitude, the primal IP method (Table 3) delivers a solution with an increasing degree of accuracy 
and virtually no loss of robustness or efficiency (Fig. 8(c) and (d)). The tangential force evolution for $\mu=10^{-1}$ is not acceptable but the forces for $\mu=10^{-3}$ are already very satisfactory and $\mu=10^{-5}$ delivers a solution that virtually overlaps with the AL solution.

Due to the large number of steps used in generating the results in Fig. 8, both the AL and the primal IP methods converge very quickly in each step such that the IP method is just as efficient as the AL one. In order to compare the degree of robustness of the two methods, two sets of tests are conducted:

1. Minimal step number: The minimal number of normal/tangential steps required for convergence at a fixed stiffness ratio is sought. The upper body is chosen to be $10^{4}$ times stiffer and the primal IP method operates with $\mu=10^{-3}$. The step number is incremented by $1 / 5$ for the normal/tangential stage during the search. The AL method requires 6 normal and 20 tangential steps for $\mathcal{N}^{6}$ and $\mathcal{N}^{5}$ discretizations. No convergence could be achieved for $\mathcal{N}^{4}$ until the search reached 100 tangential steps. Here, $\epsilon=1$ was chosen although smaller choices did not affect the results while larger choices only worsen the situation. The primal IP method requires 3 normal steps. The number of tangential steps was $15 / 20 / 25$ for $\mathcal{N}^{6} / \mathcal{N}^{5} / \mathcal{N}^{4}$. When $\mu$ is decreased to $10^{-4}$ or $10^{-5}$, only the number for $\mathcal{N}^{4}$ changes to 40 .

2. Maximal stiffness ratio: The highest possible stiffness ratio delivering convergence at a fixed number of steps is sought. The number of steps is chosen as the best primal IP performance, which is $3 / 15$ for the normal/ tangential stage. The AL method can handle a ratio of at most $10 / 1 / 1$ for $\mathcal{N}^{6} / \mathcal{N}^{5} / \mathcal{N}^{4}$ while the IP method can handle $100 / 10$ for $\mathcal{N}^{5} / \mathcal{N}^{4}$. For $\mathcal{N}^{6}$, the IP method displays no convergence problems and no deterioration in efficiency for a stiffness ratio as high as $10^{9}$ for $\mu$ as low as $10^{-5}$. The stiffness ratio and $\mu$ values are already satisfactory for a reliable simulation of a rigid indentor in contact with a highly deformable body although higher and lower values, respectively, were also achieved.

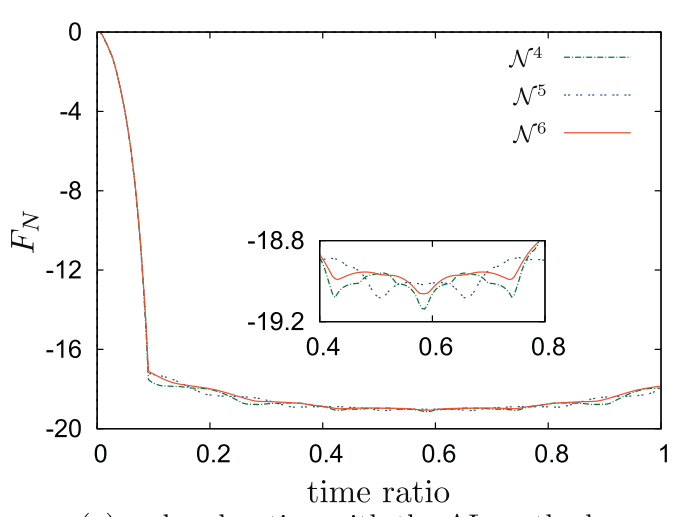

(a) order elevation with the AL method

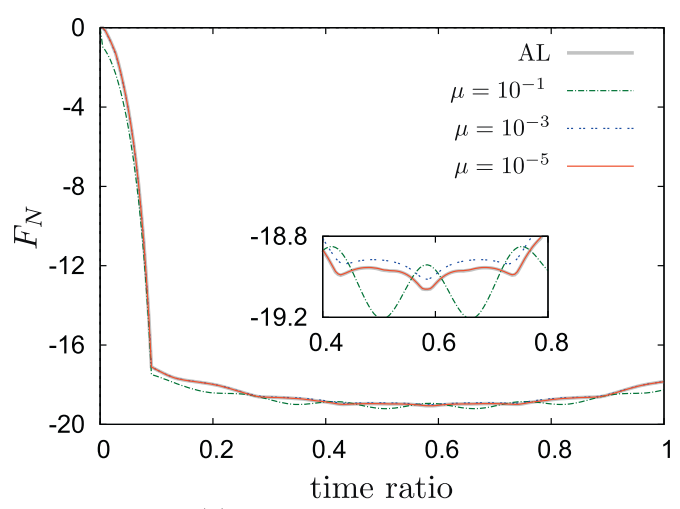

(c) barrier adjustment

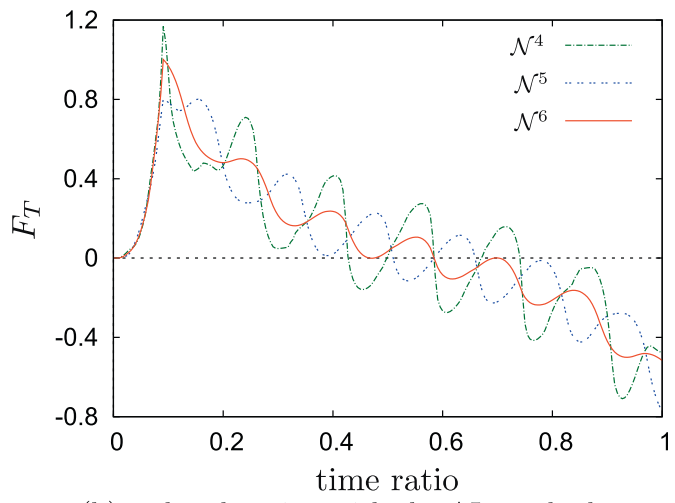

(b) order elevation with the AL method

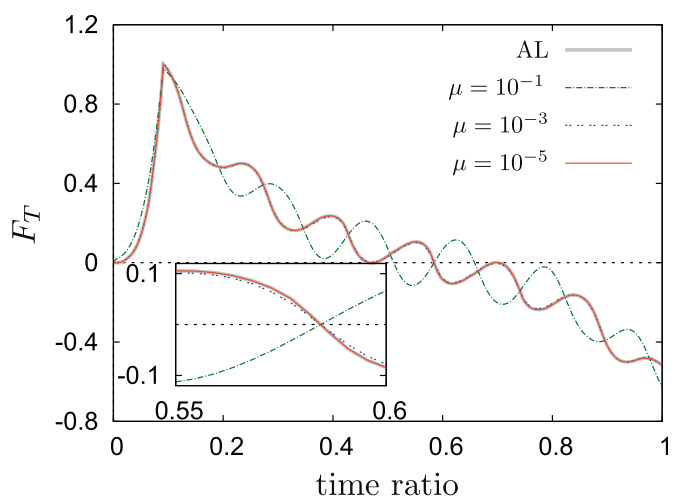

(d) barrier adjustment

Fig. 8. Problem of Fig. 7 is analyzed. (a) and (b) Compare different orders of discretization with the AL method while (c) and (d) consider the effect of the barrier parameter in the primal IP method (version 2). Here, the upper body is $10^{4}$ times stiffer. 


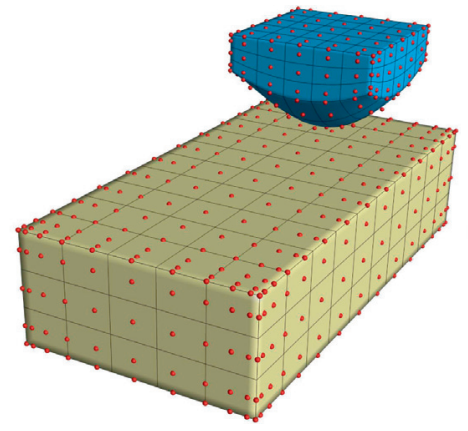

(a) discretization

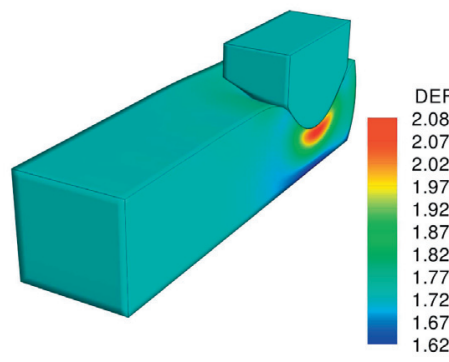

(b) end of indentation

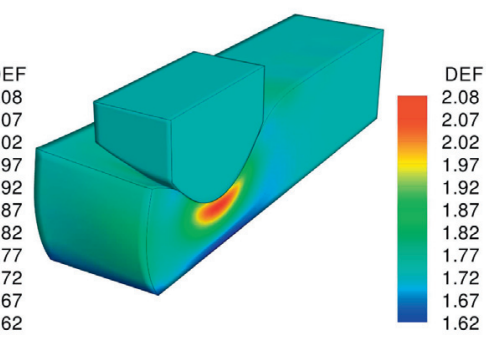

(c) end of dragging

Fig. 9. The three-dimensional version of the ironing problem of Fig. 7 is summarized, based on the same set of simulation parameters

In view of these results, one can conclude that the primal IP algorithm in Table 3 can deliver a performance that is superior to the AL method in terms of robustness. The three-dimensional version of this problem (Fig. 9) is calculated based on the same set of simulation parameters as in Fig. 7, using the primal IP method with $\mu=10^{-5}$ and $3 / 15$ normal/tangential steps. The AL method fails during the indentation stage with this step size.

\section{Conclusion}

Exterior point (EP) methods and their primal-dual extensions, notably the augmented Lagrangian (AL) approach, constitute the most widely used constraint enforcement algorithms in computational contact mechanics. In this work, an interior point (IP) algorithm was investigated. The work introduces and benefits from two major ingredients that have earlier been applied to contact mechanics problems in the context of EP methods. First, isogeometric discretizations are employed. These provide not only convenience and numerical robustness, primarily due to induced surface smoothness, but also assist in overcoming theoretical obstacles with higher-order discretizations. Second, interactively with the former, a contact discretization scheme is derived from a three-field mixed variational formulation in the spirit of the mortar method. This scheme significantly improves robustness, in addition to avoiding surface locking and passing the patch test.

As the direct counterpart of the AL approach, a primal-dual algorithm was investigated. The one adopted in this work is well-established in the optimization literature. The framework involves a number of algorithmic stages that allows flexibility for problem-specific tuning. The choices made in the present work deliver a scheme that is just as robust as the AL method for linear and nonlinear problems when the load is applied in a single step. However, the IP method was observed to be less robust for problems with multiple time/load steps. The central reason for this shortcoming is that the barrier parameter is aggressively driven to zero. On the other hand, a primal formulation was found to challenge the AL approach. One practical disadvantage of the primal formulation is that it relies on a constant-valued barrier parameter that should be judiciously chosen. Nevertheless, in all the cases tested in this work, this parameter could easily be chosen satisfactorily small without displaying any convergence problems, contrary to the penalty parameter in an EP penalty method. In fact, the primal IP method displayed a higher level of robustness than the AL method when the contacting bodies did not have the same stiffness.

Overall, it can be stated that the IP method is a viable alternative to the EP method, in particular to the popular AL approach. From a practical point of view, a potential advantage is that a smooth transition from physical interface interaction formulations to contact constraint enforcement schemes can be realized in the context of a single algorithm based on the IP method. Examples are thin-film lubrication [65] and adhesive contact problems [66]. These problems share the requirement of avoiding penetration between the interacting surfaces, which is easily handled in an IP method context but not with EP method algorithms. It may additionally be possible to combine IP and AL formulations in frictional contact where the former is used to treat normal contact in order to benefit from its observed robustness and the latter is used for tangential contact in order to benefit from established implicit time integration schemes. 


\section{Acknowledgment}

The first author acknowledges support by the European Commission under the project MultiscaleFSI (Grant No. PCIG10-GA-2011-303577).

\section{References}

[1] P. Alart, A. Curnier, A mixed formulation for frictional contact problems prone to Newton like solution methods, Comput. Methods Appl. Mech. Eng. 92 (1991) 353-375.

[2] M.H. Wright, The interior-point revolution in optimization: history, recent developments, and lasting consequences, Bull. Am. Math. Soc. (NS) 42 (2005) 39-56.

[3] J.T. Oden, S.J. Kim, Interior penalty methods for finite element approximation of the signorini problem in elastostatics, Comput. Math. Appl. 8 (1982) 33-56.

[4] D.G. Luenberger, Y. Ye, Linear and Nonlinear Programming, third ed., Springer, 2008.

[5] N. Kikuchi, J.T. Oden, Contact Problems in Elasticity: A Study of Variational Inequalities and Finite Element Methods, SIAM, 1988.

[6] G. Zavarise, P. Wriggers, E. Stein, B.A. Schrefler, Real contact mechanisms and finite element formulation - a coupled thermomechanical approach, Int. J. Numer. Methods Eng. 35 (1992) 767-785.

[7] P.W. Christensen, A. Klarbring, J.S. Pang, N. Strömberg, Formulation and comparison of algorithms for frictional contact problems, Int. J. Numer. Methods Eng. 42 (1998) 145-173.

[8] G. Tanoh, Y. Renard, D. Noll. Computational experience with an interior point algorithm for large scale contact problems. $<$ http:// www.optimization-online.org/DB_HTML/2004/12/1012.html>.

[9] G. Kloosterman, R.M.J. van Damme, A.H. van den Boogard, J. Huétnik, A geometrical-based contact algorithm using a barrier method, Int. J. Numer. Methods Eng. 51 (2001) 865-882.

[10] T. Miyamura, Y. Kanno, M. Ohsaki, Combined interior-point method and semismooth Newton method for frictionless contact problems, Int. J. Numer. Methods Eng. 81 (2010) 701-727.

[11] R. Kuc era, J. Machalová, H. Netuka, P. Z éncák, An interior-point algorithm for the minimization arising from 3D contact problems with friction, Optim. Methods Softw. http://dx.doi.org/10.1080/10556788.2012.684352 (in press).

[12] G. Stadler, Path-following and augmented Lagrangian methods for contact problems in linear elasticity, J. Comput. Appl. Math. 203 (2007) 533-547.

[13] J. Herskovits, S.R. Mazorche, A feasible directions algorithm for nonlinear complementarity problems and applications in mechanics, Struct. Multi. Optim. 37 (2009) 435-446.

[14] S.R. Mazorche, J. Herskovits, A. Canelas, G.M. Guerra, Solution of contact problems in linear elasticity using a feasible interior point algorithm for nonlinear complementarity problems, in: Proceedings of the Seventh World Congress on Structural and Multidisciplinary Optimization (COEX Seoul, 21 May-25 May 2007, Korea), 2007.

[15] J. Nocedal, S.J. Wright, Numerical Optimization, second ed., Springer, Berlin, Heidelberg, New York, 2006.

[16] J.F. Bonnans, J.C. Gilbert, C. Lemaréchal, C.A. Sagastizábal, Numerical Optimization, second ed., Springer, Berlin, Heidelberg, New York, 2006.

[17] T.J.R. Hughes, J.A. Cottrell, Y. Bazilevs, Isogeometric analysis: CAD, finite elements, NURBS, exact geometry and mesh refinement, Comput. Methods Appl. Mech. Eng. 194 (2005) 4135-4195.

[18] J.A. Cottrell, T.J.R. Hughes, Y. Bazilevs, Isogeometric Analysis, Wiley, 2009.

[19] H. Gomez, L. Cueto-Felgueroso, R. Juanes, Three-dimensional simulation of unstable gravity-driven infiltration of water into a porous medium, J. Comput. Phys. 238 (2013) 217-239.

[20] M.A. Scott, R.N. Simpson, J.A. Evans, S. Lipton, S.P.A. Bordas, T.J.R. Hughes, T.W. Sederberg, Isogeometric boundary element analysis using unstructured T-splines, Comput. Methods Appl. Mech. Eng. 254 (2013) 197-221.

[21] J.A. Evans, T.J.R. Hughes, Isogeometric divergence-conforming B-splines for the unsteady Navier-Stokes equations, J. Comput. Phys. 241 (2013) 141-167.

[22] D.J. Benson, S. Hartmann, Y. Bazilevs, M.-C. Hsu, T.J.R. Hughes, Blended isogeometric shells, Comput. Methods Appl. Mech. Eng. 255 (2013) 133-146.

[23] A.P. Nagy, S.T. Ijsselmuiden, M.M. Abdalla, Isogeometric design of anisotropic shells: optimal form and material distribution, Comput. Methods Appl. Mech. Eng. 264 (2013) 145-162.

[24] J. Lu, Isogeometric contact analysis: geometric basis and formulation of frictionless contact, Comput. Methods Appl. Mech. Eng. 200 (2011) 726-741.

[25] İ. Temizer, P. Wriggers, T.J.R. Hughes, Contact treatment in isogeometric analysis with NURBS, Comput. Methods Appl. Mech. Eng. 200 (2011) 1100-1112.

[26] P. Wriggers, Computational Contact Mechanics, second ed., Springer, Berlin, Heidelberg, New York, 2006.

[27] T.A. Laursen, Computational Contact and Impact Mechanics, first ed., Springer, Berlin, Heidelberg, New York, 2003 (corr. second printing).

[28] P. Papadopoulos, R.L. Taylor, A mixed formulation for the finite element solution of contact problems, Comput. Methods Appl. Mech. Eng. 94 (1992) 373-389.

[29] P. Papadopoulos, J.M. Solberg, A Lagrange multiplier method for the finite element solution of frictionless contact problems, Math. Comput. Model. 28 (1998) 373-384. 
[30] R.E. Jones, P. Papadopoulos, A novel three-dimensional contact finite element based on smooth pressure interpolations, Int. J. Numer. Methods Eng. 51 (2001) 791-811.

[31] M.A. Crisfield, Re-visiting the contact patch test, Int. J. Numer. Methods Eng. 48 (2000) 435-449.

[32] N. El-Abbasi, K.-J. Bathe, Stability and patch test performance of contact discretizations and a new solution algorithm, Comput. Struct. 79 (2001) 1473-1486.

[33] M.A. Puso, T.A. Laursen, A mortar segment-to-segment contact method for large deformations, Comput. Methods Appl. Mech. Eng. 193 (2004) 601-629.

[34] M.A. Puso, T.A. Laursen, A mortar segment-to-segment frictional contact method for large deformations, Comput. Methods Appl Mech. Eng. 193 (2004) 4891-4913.

[35] M.E. Matzen, T. Cichosz, M. Bischoff, A point to segment contact formulation for isogeometric, NURBS based finite elements, Comput. Methods Appl. Mech. Eng. 255 (2013) 27-39.

[36] F. Ben Belgacem, The mortar finite element method with Lagrange multipliers, Numer. Math. 84 (1999) $173-197$.

[37] M.A. Puso, A 3D mortar method for solid mechanics, Int. J. Numer. Methods Eng. 59 (2004) 315-336.

[38] B. Wohlmuth, Variationally consistent discretization schemes and numerical algorithms for contact problems, Acta Numer. 20 (2011) 569-734.

[39] P. Hild, Numerical implementation of two nonconforming finite element methods for unilateral contact, Comput. Methods Appl. Mech. Eng. 184 (2000) 99-123.

[40] K.A. Fischer, P. Wriggers, Frictionless 2D contact formulations for finite deformations based on the mortar method, Comput. Mech. 36 (2005) 226-244.

[41] S. Hüeber, B.I. Wohlmuth, A primal-dual active set strategy for non-linear multibody contact problems, Comput. Methods Appl. Mech. Eng. 194 (2005) 3147-3166.

[42] S. Hüeber, M. Mair, B.I. Wohlmuth, A priori error estimates and an inexact primal-dual active set strategy for linear and quadratic finite elements applied to multibody contact problems, Appl. Numer. Math. 54 (2005) 555-576.

[43] A. Popp, M.W. Gee, W.A. Wall, A finite deformation mortar contact formulation using a primal-dual active set strategy, Int. J. Numer. Methods Eng. 79 (2009) 1354-1391.

[44] C. Hesch, P. Betsch, A mortar method for energy-momentum conserving schemes in frictionless dynamic contact problems, Int. J. Numer. Methods Eng. 77 (2009) 1468-1500.

[45] T.W. McDevitt, T.A. Laursen, A mortar-finite element formulation for frictional contact problems, Int. J. Numer. Methods Eng. 48 (2000) 1525-1547.

[46] B. Yang, T.A. Laursen, X. Meng, Two dimensional mortar contact methods for large deformation frictional sliding, Int. J. Numer. Methods Eng. 62 (2005) 1183-1225.

[47] K.A. Fischer, P. Wriggers, Mortar based frictional contact formulation for higher order interpolations using the moving friction cone, Comput. Methods Appl. Mech. Eng. 195 (2006) 5020-5036.

[48] S. Hüeber, A. Matei, B.I. Wohlmuth, Efficient algorithms for problems with friction, SIAM J. Sci. Comput. 29 (2007) 70-92.

[49] S. Hüeber, G. Stadler, B.I. Wohlmuth, A primal-dual active set algorithm for three-dimensional contact problems with Coulomb friction, SIAM J. Sci. Comput. 30 (2008) 572-596.

[50] M.A. Puso, T.A. Laursen, J. Solberg, A segment-to-segment mortar contact method for quadratic elements and large deformations, Comput. Methods Appl. Mech. Eng. 197 (2008) 555-566.

[51] M. Tur, F.J. Fuenmayor, P. Wriggers, A mortar-based frictional contact formulation for large deformations using Lagrange multipliers, Comput. Methods Appl. Mech. Eng. 198 (2009) 2860-2873.

[52] P. Hauret, P. Le Tallec, A discontinuous stabilized mortar method for general 3d elastic problems, Comput. Methods Appl. Mech. Eng. 196 (2007) 4881-4900.

[53] İ. Temizer, A mixed formulation of mortar-based contact with friction, Comput. Methods Appl. Mech. Eng. 255 (2013) $183-195$.

[54] J.C. Simo, P. Wriggers, R.L. Taylor, A perturbed lagrangian formulation for the finite element solution of contact problems, Comput. Methods Appl. Mech. Eng. 50 (1985) 163-180.

[55] C. Hesch, P. Betsch, Isogeometric analysis and domain decomposition methods, Comput. Methods Appl. Mech. Eng. 213-216 (2012) $104-112$

[56] İ. Temizer, A mixed formulation of mortar-based frictionless contact, Comput. Methods Appl. Mech. Eng. $223-224$ (2012) 173-185.

[57] L. De Lorenzis, İ. Temizer, P. Wriggers, G. Zavarise, A large deformation frictional contact formulation using NURBS-based isogeometric analysis, Int. J. Numer. Methods Eng. 87 (2011) 1278-1300.

[58] İ. Temizer, P. Wriggers, T.J.R. Hughes, Three-dimensional mortar-based frictional contact treatment in isogeometric analysis with NURBS, Comput. Methods Appl. Mech. Eng. 209-212 (2012) 115-128.

[59] L. De Lorenzis, P. Wriggers, G. Zavarise, A mortar formulation for 3D large deformation contact using NURBS-based isogeometric analysis and the augmented Lagrangian method, Comput. Mech. 49 (2012) 1-20.

[60] J.-Y. Kim, S.-K. Youn, Isogeometric contact analysis using mortar method, Int. J. Numer. Methods Eng. 89 (2012) $1559-1581$.

[61] Y. Renard, Generalized Newtons methods for the approximation and resolution of frictional contact problems in elasticity, Comput. Methods Appl. Mech. Eng. 256 (2013) 38-55.

[62] H.Y. Benson, D.F. Shanno, An exact primal-dual penalty method approach to warmstarting interior-point methods for linear programming, Comput. Optim. Appl. 38 (2007) 371-399.

[63] H.Y. Benson, D.F. Shanno, Interior-point methods for nonconvex nonlinear programming: regularization and warmstarts, Comput. Optim. Appl. 40 (2008) 143-189. 
[64] K. Krabbenhoft, A.V. Lyamin, S.W. Sloan, P. Wriggers, An interior-point algorithm for elastoplasticity, Int. J. Numer. Methods Eng. 69 (2007) 592-626.

[65] S. Stupkiewicz, Finite element treatment of soft elastohydrodynamic lubrication problems in the finite deformation regime, Comput. Mech. 44 (2009) 605-619.

[66] R.A. Sauer, S. Li, A contact mechanics model for quasi-continua, Int. J. Numer. Methods Eng. 71 (2007) $931-962$. 